\title{
A parallel repetition theorem for entangled projection games
}

\author{
Irit Dinur* $\quad$ David Steurer $^{\dagger} \quad$ Thomas Vidick $^{\ddagger}$
}

\begin{abstract}
We study the behavior of the entangled value of two-player one-round projection games under parallel repetition. We show that for any projection game $G$ of entangled value $1-\varepsilon<1$, the value of the $k$-fold repetition of $G$ goes to zero as $O\left(\left(1-\varepsilon^{c}\right)^{k}\right)$, for some universal constant $c \geq 1$. Previously parallel repetition with an exponential decay in $k$ was only known for the case of XOR and unique games. To prove the theorem we extend an analytical framework recently introduced by Dinur and Steurer for the study of the classical value of projection games under parallel repetition. Our proof, as theirs, relies on the introduction of a simple relaxation of the entangled value that is perfectly multiplicative. The main technical component of the proof consists in showing that the relaxed value remains tightly connected to the entangled value, thereby establishing the parallel repetition theorem. More generally, we obtain results on the behavior of the entangled value under products of arbitrary (not necessarily identical) projection games.

Relating our relaxed value to the entangled value is done by giving an algorithm for converting a relaxed variant of quantum strategies that we call "vector quantum strategy" to a quantum strategy. The algorithm is considerably simpler in case the bipartite distribution of questions in the game has good expansion properties. When this is not the case, rounding relies on a quantum analogue of Holenstein's correlated sampling lemma which may be of independent interest. Our "quantum correlated sampling lemma" generalizes results of van Dam and Hayden on universal embezzlement to the following approximate scenario: two isolated parties, given classical descriptions of arbitrary bipartite states $|\psi\rangle,|\varphi\rangle$ respectively such that $|\psi\rangle \approx|\varphi\rangle$, are able to locally generate a joint entangled state $|\Psi\rangle \approx|\psi\rangle \approx|\varphi\rangle$ using an initial entangled state that is independent of their inputs.
\end{abstract}

\section{Introduction}

Two-player one-round games play an important role in many areas of theoretical computer science. They are prominent in complexity theory, where they are a powerful tool in the study of constraint satisfaction problems, and in cryptography, where they give a polyvalent abstraction in which to establish the security of many two-party primitives. They have also recently proven a very convenient framework for the study of some of the deepest issues in quantum mechanics, giving a novel viewpoint on the decades-old study of Bell inequalities, which are inequalities that must be satisfied by classical mechanics but can be violated in the presence of quantum entanglement.

* Department of Computer Science and Applied Math, The Weizmann Institute, Israel. Research supported by ERC grant number 239985. Part of the work was done while the author was visiting MIT supported by NSF Contract CCF-1018064, and by Simons Investigator Award of Shafi Goldwasser.

${ }^{\dagger}$ Departement of Computer Science, Cornell University. Part of this work was done at Microsoft Research New England.

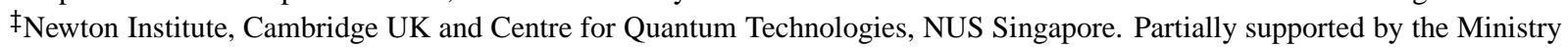
of Education, Singapore under the Tier 3 grant MOE2012-T3-1-009. Part of this work was completed while the author was at MIT, supported by the National Science Foundation under Grant No. 0844626. 
A game $G$ is specified by a probability distribution $\mu$ on pairs of questions $(u, v) \in \mathcal{U} \times \mathcal{V}$ to the players, and an acceptance criterion $V \subseteq \mathcal{A} \times \mathcal{B} \times \mathcal{U} \times \mathcal{V}$ which states, for every possible pair of questions $(u, v)$, which pairs of answers $(a, b) \in \mathcal{A} \times \mathcal{B}$ are valid. The most basic quantity associated to a game is its value: the maximum success probability of two cooperating, but spatially isolated, players in the game. Remarkably, the precise definition of the value depends on the physical interpretation that is given to the condition of spatial isolation. Under classical theory, isolated players are fully described by the functions that each apply to their respective question in order to determine their answer, and this interpretation leads to the classical value VAL of the game. In contrast, in quantum theory isolated players are allowed any set of strategies that can be implemented by performing local measurements on a shared entangled state. The resulting value is called the entangled value and denoted $\operatorname{vAL}^{*}(G)$. Clearly for every game it holds that $\mathrm{VAL} \leq \mathrm{VAL}^{*}$, and it is the discovery of Einstein, Podolsky and Rosen [EPR35] (formalized by Bell [Bel64], simplified by Clauser et al. [CHSH69] and experimentally verified by Aspect et al. [AGR81]) that there exists games for which the inequality is strict; indeed there are families of games $\left(G_{n}\right)$ for which $\operatorname{vAL}\left(G_{n}\right) \rightarrow 0$ but $\operatorname{VAL}^{*}\left(G_{n}\right)=1$ [Raz98, Ara02]. One can go even further and consider the non-signaling value $\operatorname{VAL}^{n s}$, which corresponds to players allowed to reproduce any bipartite correlations that do not imply signaling between their isolated locations. Here again $\mathrm{VAL}^{*} \leq \mathrm{VAL}^{n s}$, and there are games, such as the CHSH game [CHSH69], for which the inequality is strict.

One of the most fundamental questions one may ask about two-player games is that of the behavior of the value under product. Given games $G$ and $H$, their product $G \otimes H$ is defined as follows: the question and answer sets are the cartesian product of those from $G$ and $H$; the distribution on questions is the product of the distributions, and the acceptance criterion the AND of those of $G$ and of $H$. How does the value of $G \otimes H$ relate to that of $G$ and $H$ ? While it is clear that each of the three values defined above satisfies $\operatorname{vaL}(G \otimes$ $H) \geq \operatorname{VAL}(G) \operatorname{vaL}(H)$, the converse, although intuitive, does not hold in most nontrivial scenarios. In particular, simple constructions of games $G$ are known such that $\operatorname{vAL}(G \otimes G)=\operatorname{VAL}(G)<1$ [FL92]; similar constructions exist for VAL $\left[\right.$ [CSUU08] and VAL ${ }^{\text {ns }}$ [KR10].

In spite of these examples, one may still ask for the behavior of $\operatorname{VAL}\left(G^{\otimes k}\right)$, for "large" values of $k$. This is known as the parallel repetition question: given a game $G$ such that $\operatorname{VAL}(G)<1$, does there exist an $\alpha<1$ such that $\operatorname{VAL}\left(G^{\otimes k}\right) \leq \alpha^{k}$ ? If so, what is the dependence of $\alpha$ on $1-\operatorname{VAL}(G)$ ? Answering this question is of importance for many of the applications of two-player games. In cryptography, parallel repetition is a basic primitive using which one may attempt to amplify the security guarantees of a given protocol; in the study of Bell inequalities it can be used e.g. to amplify gaps between the quantum and non-signaling values; in complexity theory it is at the heart of hardness amplification.

For the case of the classical value, a long sequence of works [Ver94, Fei91, FK00] over the course of a decade led to the breakthrough by Raz [Raz98], who was the first to provide a positive answer for general games: Raz showed that one can always take $\alpha=\left(1-\left(1-\operatorname{VAL}(G)^{c}\right)^{d \log |\mathcal{A} \times \mathcal{B}|}\right.$, where $c, d$ are universal constants. Subsequent work focused on obtaining the best possible value for $c$ (the best known for general games is $c=3$ [Hol09]) and on removing the dependence on the size of the answer alphabet for specific classes of games [Ra008, $\mathrm{BRR}^{+}$09, RR12]. For the case of the no-signaling value, Holenstein showed one can always take $\alpha=1-\operatorname{VAL}(G)^{2}$ [Hol09].

In contrast, for the case of the entangled value in spite of its importance the question is very poorly understood. Strong results are known for some very special classes of games such as XOR games [CSUU08], for which repetition is exact (one can take $\alpha=\mathrm{VAL}^{*}(G)$ ) and unique games [KR10] (for $\alpha=1-C(1-$ $\left.\operatorname{VAL}^{*}(G)\right)^{2}$, where $C>0$ is a universal constant). However, both these results, as well as related results motivated by cryptographic applications [HR09], rely on the formulation of the entangled value as a semidefinite program, a characterization that is not believed to extend to more general games. Additional results 
are known but they only apply to specific games often originating from cryptography [MPA11, TFKW13]. The most general results known to date come from [KV11], where it is shown that a specific type of repetition inspired by work of Feige and Kilian [FK00], in which the original game is mixed with "consistency" and "free" games, reduces the entangled value at a polynomial rate: provided $\operatorname{VAL}^{*}(G)<1$, the value $\operatorname{VAL}^{*}\left(G^{F K-\otimes k}\right)$ of $k$ "Feige-Kilian" repetitions of $G$ behaves as $((1-\operatorname{VAL}(G)) k)^{-c}$ for some small $c>0$.

A recent work of Dinur and Steurer [DS13] introduces a new approach to the parallel repetition question, focused on the case of projection games. A projection game is one in which the referee's acceptance criterion has a special form: for any pair of questions $(u, v)$, any answer $b$ from the second player determines at most one valid answer $a=\pi_{u v}(b)$ for the first player. Projection games are the most interesting and widelystudied type of games. The standard transformation from 3SAT to a two-player game naturally results in a projection game: one player is asked for an assignment to a random clause, and the other is asked for an assignment to one of its three variables. This simple transformation easily generalizes to convert any constraint satisfaction problem or general two-player game $G$ into a projection game $G^{\prime}$, while essentially preserving the value: $1-\operatorname{VAL}\left(G^{\prime}\right)=\Theta(1-\operatorname{vAL}(G))$ (see Claim 3). In particular, if one is only interested in "amplifying the gap" between $\operatorname{VAL}(G)=1$ and $\operatorname{vaL}(G)<1$ one can first map $G$ to $G^{\prime}$ and then consider the parallel repetition of $G^{\prime}$ itself, and this justifies the predominant role played by projection games in classical complexity theory. The transformation, however, may decrease the entangled value arbitrarily whenever the optimal strategy for the players requires the use of entanglement (though it can never increase it by too much; see Claim 3 for precise bounds). Nevertheless, many of the games studied in quantum information, such as the CHSH game [CHSH69] or the Magic Square game [Ara02] are projection games.

The approach of [DS13] is based on the introduction of a relaxation of the game value, denoted VAL . This relaxation can be defined for any game (we give the definition in Section 1.2 below), and it is perfectly multiplicative. Moreover, for the case of projection games $\mathrm{VAL}_{+}$turns out to remain closely related to VAL, thus giving a parallel repetition theorem. Although such a theorem already follows from Raz's general result [Raz98], this arguably simpler approach matches the best parameters currently known [Rao08] (which are known to be optimal [Raz08]). In addition, it yields new results for repetitions of games with small value and the case of few repetitions, which has implications for the approximability of the LABEL COVER and SET COVER problems.

\subsection{Our results}

We extend the analytical framework introduced in [DS13] to the case of the entangled value VAL* As a consequence we obtain the following main theorem on the parallel repetition of the entangled value of projection games.

Theorem 1. There exists constants $c, C>0$ such that the following holds. For any projection game G,

$$
\operatorname{VAL}^{*}\left(G^{\otimes k}\right) \leq\left(1-C\left(1-\operatorname{VAL}^{*}(G)\right)^{c}\right)^{k / 2}
$$

Although we do not attempt to fully optimize the constant $c$, values that come out of our proof are $c \leq 4$ for the case of expanding games (see definition in Section 2.2) and $c \leq 12$ for arbitrary projection games.

Parallel repetition results for the classical value were originally motivated by the study of multi-prover interactive proofs [FRS88], and our result is likewise applicable to the study of classes of multi-prover interactive proofs with entangled provers. For instance, it enables soundness amplification in some specific cases. Letting $\mathrm{MIP}_{1, \mathrm{~s}}^{\mathrm{er}}(2)$ denote the class of languages having 2-prover 1-round interactive proofs in which completeness $c=1$ holds with unentangled provers, but soundness $s$ holds even against provers allowed to 
share entanglement, then Theorem 1 implies that $\operatorname{MIP}_{1, s}^{\text {er }}(2)=\operatorname{MIP}_{1,2^{- \text {poly }}}^{\text {er }}(2)$ for any $s<1-$ poly $^{-1}(n)$. This is because any protocol in $\mathrm{MIP}_{1, S}^{\mathrm{er}}(2)$ can be put into a form where the verifier's test is a projection constraint by following the reduction already discussed above, and described in Claim 3, this will preserve both perfect completeness (for classical strategies) and soundness bounded away from 1 (for quantum strategies). Prior to our work it was not known how to amplify soundness to exponentially small without increasing the number of rounds of interaction. It follows from [IV12, Vid13] that $\mathrm{MIP}_{1,1-\text { poly }^{-1}}^{\mathrm{er}}(3)=\mathrm{NEXP}$, but very little is known about the 2-prover class $\operatorname{MIP}_{1, s}^{\mathrm{er}}(2)$.

We believe that our results, however, should find applications to a much wider range of problems. Going beyond the application to the parallel repetition question, our main contribution is the development of a precise framework in which general questions about the behavior of the value under product can be studied. This framework constitutes a comprehensive extension of the one introduced in [DS13] for the study of the classical value: as in [DS13], we introduce a relaxation $\mathrm{VAL}_{+}^{*}$ of the entangled value, prove that it is perfectly multiplicativity, and show that it remains closely related to $\mathrm{VAL}^{*}$. We find it remarkable that the framework from [DS13], introduced in a purely classical context, would find such a direct, if nontrivial, extension to the case of the entangled value. We hope that the tools developed in this extension will find further applications to the proof of product theorems in areas ranging from cryptography to communication complexity. Even though at a technical level the setting can appear quite different, some of the ideas put forth here could also prove useful to further removed areas such as the multiplicativity conjecture for the minimum output entropy of quantum channels [AHW00, HW08, Has09].

We turn to a more detailed explanation of our framework, hoping to highlight precisely those tools and ideas that may find further application.

\subsection{Proof sketch}

In order to explain our approach it is useful to first review the framework introduced in [DS13] for the study of the classical value.

Classical strategies. The starting point in [DS13] consists in viewing games as operators acting on the space of strategies. In this language a strategy is simply a vector $|f\rangle$ of non-negative reals indexed by pairs $(u, a)$ of possible questions and answers: $f(u, a)$ is the probability that the strategy decides to provide answer $a$ to question $u$. To any game one can associate a matrix $G$ such that, formally, the success probability of strategies $(|f\rangle,|g\rangle)$ for the players precisely evaluates to the vector-matrix-vector product $\langle f|G| g\rangle$. The value of the game is then the norm of $G$ when viewed as an operator from the appropriately normed spaces of strategies.

The first crucial step taken in [DS13] consists in relaxing the value of a game $G$ to the value of the square $G^{\dagger} G$ of the game (this notation will be made precise in Section 2.2); we will denote the latter by $\|G\|_{\square}$. In the square of a game $G$, the referee first samples a question $u$ for the first player as in $G$. He then independently samples two questions $v$ and $v^{\prime}$ for the second player according to the conditional distribution. The players in $G^{\dagger} G$ are sent $v$ and $v^{\prime}$ respectively. They have to provide answers $b$ and $b^{\prime}$ such that there exists an $a$ such that both $(a, b)$ is a valid answer to $(u, v)$ in $G$, and $\left(a, b^{\prime}\right)$ is a valid answer to $\left(u, v^{\prime}\right)$. Note that now $G^{\dagger} G$ treats both players symmetrically, and it turns out that we may always assume that they both apply the same strategy. For the special case of projection games it is not hard to show that the value of the game and that of its square are quadratically related:

$$
\operatorname{VAL}(G)^{2} \leq\|G\|_{\square} \leq \operatorname{vAL}(G) .
$$


Indeed, using the algebraic language introduced above, the first inequality follows from the Cauchy-Schwarz inequality and the second is an easy observation.

The second step consists in observing that the application of the operator corresponding to the product $G \otimes H$, where $G$ and $H$ are arbitrary projection games, can be decomposed as a product $(G \otimes I) \cdot(I \otimes H)$. Starting with a strategy $|f\rangle$ for $G \otimes H$, the result of applying $(I \otimes H)$ to $|f\rangle$ is a new vector which no longer satisfies the strict normalization requirements of strategies. Understanding the new normalization leads to a further relaxation of $\|G\|_{\square}$, denoted $\operatorname{VAL}_{+}(G)$, in which the optimization is performed over the appropriate notion of "vector strategies", which intuitively are vectors that can be obtained by applying game operators to strategies. With the correct definition, it is easy to show that

$$
\|G \otimes H\|_{\square}^{2} \leq \operatorname{vAL}_{+}(G) \cdot\|H\|_{\square}^{2} .
$$

The third and last step, which constitutes most of the technical work in [DS13], consists in showing that $\operatorname{VAL}_{+}(G)$ is a good approximation to $\|G\|_{\square}$. This is done using a rounding procedure, by which a vector strategy associated with a large $\mathrm{VAL}_{+}$is mapped back to an actual strategy for the square game that also has a high value, thus serving as a witness for the value $\|G\|_{\square}$ being large as well. Altogether we get a bound on the value of $G \otimes H$ as a product of a bound on the value of $G$ and a bound on the value of $H$. Repeated application of (2) then leads to the following chain of inequalities

$$
\operatorname{VAL}\left(G^{\otimes k}\right)^{2} \leq\left\|G^{\otimes k}\right\|_{\square}^{2} \leq \operatorname{VAL}_{+}(G) \cdot\left\|G^{\otimes k-1}\right\|_{\square}^{2} \leq \cdots \leq \operatorname{VAL}_{+}(G)^{k} \approx \operatorname{VAL}(G)^{k},
$$

proving the parallel repetition theorem.

Quantum strategies. Our goal now is to extend the above sketch to the case of the entangled value VAL* There is good reason for optimism. In contrast to most classical proofs used in the study of classical twoplayer games (such as those that go into Dinur's proof of the PCP theorem [Din07], or earlier approaches to parallel repetition [Ver94, FK00, Raz98]), which are often information-theoretic or combinatorial in nature, the analytic (one could say linear-algebraic) framework introduced in [DS13] seems much better suited a priori to an extension to the quantum domain. Indeed, quantum strategies themselves are objects that live in $d$-dimensional complex vector space: instead of a vector of non-negative reals (describing the probability of answering $a$ to question $u$, for every possible $u$ and $a$ ), a strategy is now a vector $|A\rangle$ of $d$-dimensional positive semidefinite matrices $A_{u}^{a}$ that describe the measurement to be performed upon receiving any question $u$. The normalization condition is $\sum_{a} A_{u}^{a}=\operatorname{Id}$ for every $u$, a constraint dictated by the formalism of measurements in quantum mechanics.

At an abstract level, going from the classical to the entangled value thus solely requires us to think of the game $G$ as an operator acting on a bigger space of strategies, "enlarging" the non-negative reals to the space of $d$-dimensional positive semidefinite matrices. This operation is easily realized by "tensoring with identity", $G \rightarrow G \otimes \mathrm{Id}_{\mathbb{C}^{d}}$. This said, extending each of the steps outlined above nevertheless raises a number of challenges unique to the quantum setting, in which far more than in the classical case the strength of strategies usually requires them to be studied in conjunction with the entanglement that enables their unique form of correlation.

The first step consists in obtaining an analogue of (1). As in the classical case the second inequality is easy, and follows by observing that, if $|A\rangle$ is a quantum strategy in $G^{\dagger} G$ then $(G \otimes \operatorname{Id})|A\rangle$ is a valid strategy for the first player in $G$ (this notation will be made precise in Section 2.2) The first inequality in (1) is slightly more subtle. Although it can be proven directly by applying a suitable matrix version of the Cauchy-Schwarz inequality, we note that it can also be proven using known properties of a widely used 
construct in quantum information theory, the pretty-good measurement (PGM) [HW94, $\mathrm{HJS}^{+}$96]. As it turns

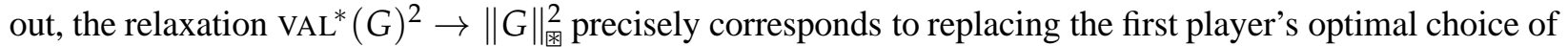
strategy in $G$ by a near-optimal choice obtained from the pretty-good-measurement. As a consequence, (1) extends verbatim:

$$
\operatorname{VAL}^{*}(G)^{2} \leq\|G\|_{\text {番 }}^{2} \leq \operatorname{VAL}^{*}(G) .
$$

Next we need to find an appropriate notion of vector strategy and corresponding relaxed value $\mathrm{VAL}_{+}^{*}$. Here we are helped by the "operational" interpretation of a vector strategy as the result of the application a game operator to a strategy meant for the product of several games. With the suitable generalization of the definition of classical vector strategies (see Definition 9) we also obtain an analogue of (2) for VAL $L_{+}^{*}$ :

$$
\|G \otimes H\|_{\circledast}^{2} \leq \operatorname{VAL}_{+}^{*}(G) \cdot\|H\|_{\text {ख }}^{2} .
$$

Finally, and most arduous, is to relate the relaxation $\mathrm{VAL}_{+}^{*}$ back to the value of the square game, $\|G\|_{\text {承. }}^{2}$. In the classical case this involves rounding vector to actual strategies. In the quantum case rounding has to be performed synchronously by the players, and will necessarily involve the use of an entangled state. Intuitively, upon receiving their respective questions in $G$ the players need to initialize themselves in an entangled state that corresponds to the "post-measurement state" that they would be in, conditioned on having given a particular pair of answers to a given pair of questions in the game $H$ from which the vector strategy is derived (recall that, informally, vector strategies are the result of applying a game operator to a strategy meant for the product of two or more distinct games).

In case the bipartite distribution of questions in the game $G$ has good expansion properties we can show that this conditioned state is roughly the same regardless of the questions in $G$, so there is a way for players to renormalize their measurements and proceed. For the non-expanding case the states can differ significantly from question to question. Nevertheless, we can show that based on their respective questions the players are able to agree on classical descriptions of two close states $|\psi\rangle \approx|\varphi\rangle$ that they respectively wish to be in.

At this point an interesting component of our proof is a new "quantum correlated sampling" lemma which allows the players to generate a joint entangled state $|\Psi\rangle \approx|\psi\rangle \approx|\varphi\rangle$ from an initial shared universal "embezzlement state" $\mathrm{vH03}$ independent of $|\varphi\rangle$ or $|\psi\rangle$, without any communication. The lemma can be seen as a quantum variant of Holenstein's correlated sampling lemma [Hol09], as well as a "robust" extension of the results of van Dam and Hayden on universal embezzlement states [vH03].

All steps having been extended, we obtain a direct generalization of the chain of inequalities (3) to the case of entangled strategies:1

$$
\operatorname{VAL}^{*}\left(G^{\otimes k}\right)^{2} \leq\left\|G^{\otimes k}\right\|_{\circledast}^{2} \leq \operatorname{VAL}_{+}^{*}(G) \cdot\left\|G^{\otimes k-1}\right\|_{\circledast}^{2} \leq \cdots \leq \operatorname{VAL}_{+}^{*}(G)^{k} \approx\left(\operatorname{VAL}^{*}(G)\right)^{k} .
$$

\subsection{Additional related work}

Although few general results are known, the question of the behavior of the entangled value of a twoplayer game or protocol under parallel repetition arises frequently. It plays an important role in recent results on device-independent quantum key distribution [HR09, MPA11] and related cryptographic primitives [TFKW13]. The latter work considers parallel repetition of a game with quantum messages, a setting which is also the focus of [CJPP11]. The approach of [CJPP11] builds upon [JPPG ${ }^{+} 10$ ], who relate the (classical) value of a two-player one-round game to the norm of the game when viewed as a tensor on the

\footnotetext{
${ }^{1}$ We note however that the approximate equality $\operatorname{vAL}_{+}^{*}(G) \approx \operatorname{vAL}^{*}(G)$ that we obtain in the quantum case, although it suffices for our application to parallel repetition, is weaker than the one from [DS13]. In particular, it is probably not tight.
} 
space $\ell_{\infty}\left(\ell_{1}\right) \otimes \ell_{\infty}\left(\ell_{1}\right)$. This is similar to our starting point of viewing games as operators acting on strategies, except that it considers the game as a bilinear form rather than an operator; the two points of view are equivalent. This perspective enables the authors to leverage known results on the study of tensor norms in Banach space (resp. operator space) theory to derive results on the classical (resp. entangled) value. To the best of our knowledge this connection has not led to an alternative approach to proving parallel repetition for general classes of games, although partial results were obtained in [CJPP11] for the special case of the entangled value of rank-one quantum games.

\subsection{Open questions}

We briefly mention several interesting open questions. There still does not exist any parallel repetition result that applies to the entangled value of general, non-projection two-player one-round games, and it would be interesting to investigate whether our techniques could lead to (even relatively weak) results in the general setting. The case of three players is also of interest, and no non-trivial parallel repetition results are known either in the classical or quantum setting. In fact, the closely related question of XOR repetition of threeplayer games is known to fail dramatically even for the classical value [BBLV12].

Organization of the paper. We start with some important preliminaries in Section 2 There we introduce the representation of games and strategies that is used throughout the remainder of the paper. In Section 3 we introduce the two relaxations of the entangled value sketched in the introduction and give a more detailed overview of our proof. In Section 4 we prove the main technical component of our work, the relation

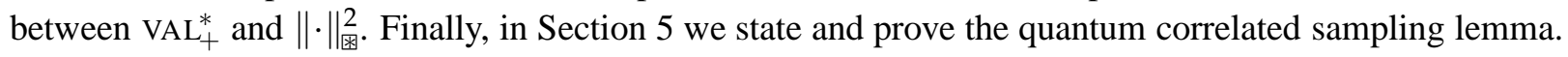

\section{Preliminaries}

\subsection{Notation}

We identify $\mathcal{L}\left(\mathbb{C}^{d^{\prime}}, \mathbb{C}^{d}\right)$, the set of linear operators from $\mathbb{C}^{d^{\prime}}$ to $\mathbb{C}^{d}$, with the set of $d \times d^{\prime}$ matrices with complex entries: if $X \in \mathcal{L}\left(\mathbb{C}^{d^{\prime}}, \mathbb{C}^{d}\right)$ then its matrix has entries $X_{a, b}=\langle a|X| b\rangle$, where $|a\rangle$, $|b\rangle$ range over the canonical basis for $\mathbb{C}^{d}, \mathbb{C}^{d^{\prime}}$ respectively, and we use the bra-ket notation to denote column vectors $|b\rangle$ and row vectors $\langle a|=(|a\rangle)^{\dagger}$, where + denotes the conjugate-transpose. We also write $\mathcal{L}\left(\mathbb{C}^{d}\right)$ for $\mathcal{L}\left(\mathbb{C}^{d}, \mathbb{C}^{d}\right)$. The space $\mathcal{L}\left(\mathbb{C}^{d^{\prime}}, \mathbb{C}^{d}\right)$ is a Hilbert space for the inner product $\langle A, B\rangle:=\operatorname{Tr}\left(A^{\dagger} B\right)$. We let $\|X\|_{\infty}$ be the operator norm of $X$, its largest singular value. A state $|\Psi\rangle \in \mathbb{C}^{d}$ is a vector with norm 1 .

\subsection{Games and strategies}

Definitions. A two-player game is specified by question sets $\mathcal{U}$ and $\mathcal{V}$, answer sets $\mathcal{A}$ and $\mathcal{B}$, a distribution $\mu$ on $\mathcal{U} \times \mathcal{V}$, and an acceptance rule $V \subseteq \mathcal{A} \times \mathcal{B} \times \mathcal{U} \times \mathcal{V}$. The game may also be thought of as a bipartite constraint graph, with vertex sets $\mathcal{U}$ and $\mathcal{V}$, edge weights $\mu(u, v)$, and constraints $V(a, b, u, v)=1$ on each edge $(u, v)$. We will write $\mu_{L}$ for the marginal distribution of $\mu$ on $\mathcal{U}$, and $\mu_{R}$ its marginal on $\mathcal{V}$. (We omit the subscripts $L$ and $R$ when they are clear from context.) We also often write $v \sim u$ to mean that $v$ is distributed according to the conditional distribution $\mu(v \mid u)=\mu(u, v) / \mu_{L}(u)$. The size of $G$ is defined as $|\mathcal{U}||\mathcal{V}||\mathcal{A}||\mathcal{B}|$

In this paper we focus on projection games, which are games for which the acceptance rule $V$ is such that for every $(u, v, b) \in \mathcal{U} \times \mathcal{V} \times \mathcal{B}$ there is at most one $a \in \mathcal{A}$ such that $V(a, b, u, v)=1$. Equivalently, for every edge $(u, v)$ the associated constraint is a projection constraint $\pi_{u, v}: \mathcal{B} \rightarrow \mathcal{A}$ such that $\pi_{u, v}(b)$ is 
the unique $a$ such that $V(a, b, u, v)=1$ if it exists, and a special "fail" symbol $\perp$ otherwise. When the edge $(u, v)$ is clear from context we will write $b \rightarrow a$ to mean that $\pi_{u v}(b)=a$. We also write $b \leftrightarrow b^{\prime}$ to mean that there exists an $a, b \rightarrow a$ and $b^{\prime} \rightarrow a$.

Given a projection game $G$, let $H$ be the weighted adjacency matrix associated with the square of $G$ : $H$ is the $|\mathcal{V}| \times|\mathcal{V}|$ matrix whose $\left(v, v^{\prime}\right)$-th entry equals $\mu\left(v, v^{\prime}\right):=\sum_{u} \mu(u) \mu(v \mid u) \mu\left(v^{\prime} \mid u\right)$. Let $D$ be the diagonal matrix with the degrees $\mu_{R}(v)$ on the diagonal, and $L:=\mathrm{Id}-D^{-1 / 2} H D^{-1 / 2}$ the normalized Laplacian associated with the square of $G$. We say that a family of games $\left(G_{n}\right)$, where $G_{n}$ has size $n$, is expanding if the second smallest eigenvalue of $L_{n}=L\left(G_{n}\right)$ is at least a positive constant independent of $n$.

Projection games as operators. Let $G$ be a two-player projection game. We will think of $G$ as a linear operator $G: \mathbb{C}^{|\mathcal{V}|} \otimes \mathbb{C}^{|\mathcal{B}|} \rightarrow \mathbb{C}^{|\mathcal{U}|} \otimes \mathbb{C}^{|\mathcal{A}|}$ defined as follows:

$$
G:=\sum_{u, v} \mu(v \mid u) \sum_{a, b \rightarrow a}|u\rangle\langle v|\otimes| a\rangle\langle b| \in \mathcal{L}\left(\mathbb{C}^{|\mathcal{V}|} \otimes \mathbb{C}^{|\mathcal{B}|}, \mathbb{C}^{|\mathcal{U}|} \otimes \mathbb{C}^{|\mathcal{A}|}\right) .
$$

In other words, for $|B\rangle \in \mathbb{C}^{|\mathcal{V}|} \otimes \mathbb{C}^{|\mathcal{B}|}$, let $B_{v}^{b}=\langle v, b \mid B\rangle$ denote the value of $B$ at point $v, b$. Then $(G B)_{u}^{a}=$ $\sum_{v} \mu(v \mid u) \sum_{b \rightarrow a} B_{v}^{b}$. Note that here we adopted the convention that questions $u \in \mathcal{U}$ are summed over, whereas questions $v \in \mathcal{V}$ are weighted by the corresponding conditional probability $\mu(v \mid u)$.

Classical strategies. The actions of players in a game $G$ give rise to a "probabilistic assignment", a collection of probability distributions $\{p(a, b \mid u, v)\}$ such that, for any pair of questions $(u, v), p(\cdot, \cdot \mid u, v)$ is a probability distribution on pairs of answers to those questions. We may also represent $p$ as the rectangular $|\mathcal{U}||\mathcal{A}| \times|\mathcal{V}||\mathcal{B}|$ matrix whose $((u, a),(v, b))$-th entry is $p(a, b \mid u, v)$. The value achieved by $p$ in the game is defined as

$$
\operatorname{vAL}(G, p):=\operatorname{Tr}_{\mu}(G p)=\sum_{u} \mu(u) \sum_{a} \sum_{v} \mu(v \mid u) \sum_{b \rightarrow a} p(a, b \mid u, v)
$$

where here we introduced a trace $\operatorname{Tr}_{\mu}$ on the set of all $X \in \mathcal{L}\left(\mathbb{C}^{|\mathcal{U}|} \otimes \mathbb{C}^{|\mathcal{A}|}\right)$ by defining

$$
\operatorname{Tr}_{\mu}(X)=\sum_{u} \mu(u) \sum_{a} X_{(u, a),(u, a)} .
$$

In cases of interest the family of distributions $\{p(a, b \mid u, v)\}$ is not arbitrary, but has a bipartite structure which reflects the bipartite nature of the game. Classical strategies correspond to the case when $p(a, b \mid u, v)=f(a \mid u) g(b \mid v)$ for functions $f(\cdot \mid u): \mathcal{A} \rightarrow\{0,1\}$ and $g(\cdot \mid v): \mathcal{B} \rightarrow\{0,1\}$ taking the value 1 exactly once. The functions $f$ and $g$ may be represented as vectors

$$
|f\rangle=\sum_{u, a} f(a \mid u)|u\rangle|a\rangle \in \mathbb{C}^{|\mathcal{U}|} \otimes \mathbb{C}^{|\mathcal{A}|} \quad \text { and } \quad|g\rangle=\sum_{v, b} g(b \mid v)|v\rangle|b\rangle \in \mathbb{C}^{|\mathcal{V}|} \otimes \mathbb{C}^{|\mathcal{B}|}
$$

respectively. $p$ is then the rank-one matrix $p=|g\rangle\langle f|$, and we may express the value as

$$
\operatorname{vAL}(G, p)=\operatorname{Tr}_{\mu}(G p)=\langle f, G g\rangle_{\mu_{L}}=\sum_{u} \mu_{L}(u) \sum_{v} \mu(v \mid u) \sum_{a} \sum_{b \rightarrow a} f(a \mid u) g(b \mid v),
$$

where the inner product $\langle\cdot, \cdot\rangle_{\mu_{L}}$ is defined on $\left(\mathbb{C}^{\mathcal{U}} \otimes \mathbb{C}^{\mathcal{A}}\right) \times\left(\mathbb{C}^{\mathcal{U}} \otimes \mathbb{C}^{\mathcal{A}}\right)$ by

$$
\langle f, g\rangle_{\mu_{L}}:=\sum_{u} \mu_{L}(u) \sum_{a} f(u, a) g(u, a) .
$$

We may similarly define an inner product $\langle\cdot, \cdot\rangle_{\mu_{R}}$ on $\left(\mathbb{C}^{\mathcal{V}} \otimes \mathbb{C}^{\mathcal{B}}\right) \times\left(\mathbb{C}^{\mathcal{V}} \otimes \mathbb{C}^{\mathcal{B}}\right)$, and we will omit the subscripts $L, R$ when they are clear from context. Given a game matrix $G$, we define its adjoint $G^{\dagger}$ as the unique matrix such that $\langle f, G g\rangle_{\mu_{L}}=\left\langle G^{\dagger} f, g\right\rangle_{\mu_{R}}$ for all $f \in \mathbb{C}^{\mathcal{U} \times \mathcal{A}}$ and $g \in \mathbb{C}^{\mathcal{V} \times \mathcal{B}}$. Formally, if $G=\sum_{u, v} \mu(v \mid u) \sum_{b \rightarrow a}|u\rangle\langle v|\otimes| a\rangle\langle b|$ then $G^{+}=\sum_{u, v} \mu(u \mid v) \sum_{b \rightarrow a}|v\rangle\langle u|\otimes| b\rangle\langle a|$. 
Quantum strategies. Next we consider quantum strategies. A quantum strategy is specified by measurements $\left\{A_{u}^{a}\right\}_{a}$ for every $u$ and $\left\{B_{v}^{b}\right\}_{b}$ for every $v$, where in general a measurement is any collection of positive semidefinite operators that sum to identity. For any state $|\Psi\rangle .2$ this strategy gives rise to the family of distributions

$$
p_{|\Psi\rangle}(a, b \mid u, v):=\left\langle\Psi\left|\overline{A_{u}^{a}} \otimes B_{v}^{b}\right| \Psi\right\rangle \mid 3
$$

This formula, dictated by the laws of quantum mechanics, corresponds to the probability that the players obtain outcomes $a, b$ when performing the measurements $\left\{A_{u}^{a}\right\},\left\{B_{v}^{b}\right\}$ on their respective share of $|\Psi\rangle$. One can check that positive semidefiniteness of the measurement operators together with the "sum to identity" condition imply that $p_{|\Psi\rangle}(\cdot, \cdot \mid u, v)$ is a well-defined probability distribution on $\mathcal{A} \times \mathcal{B}$. To a quantum strategy we associate vectors

$$
|A\rangle=\sum_{u, a}|u\rangle|a\rangle \otimes A_{u}^{a} \in \mathbb{C}^{|\mathcal{U}|} \otimes \mathbb{C}^{|\mathcal{A}|} \otimes \mathcal{L}\left(\mathbb{C}^{d}\right) \quad \text { and } \quad|B\rangle=\sum_{v, b}|v\rangle|b\rangle \otimes B_{v}^{b} \in \mathbb{C}^{|\mathcal{V}|} \otimes \mathbb{C}^{|\mathcal{B}|} \otimes \mathcal{L}\left(\mathbb{C}^{d}\right) .
$$

(Note that these definitions reduce to classical strategies whenever $d=1$.) To express the success probability of this strategy in a game $G$ we extend the definition of the inner product $\langle\cdot, \cdot\rangle_{\mu}$ as follows.

Definition 2 (Extended Inner Product). We define the extended inner product

$$
\langle\cdot, \cdot\rangle_{\mu_{L}}: \mathbb{C}^{|\mathcal{U}|} \otimes \mathbb{C}^{|\mathcal{A}|} \otimes \mathcal{L}\left(\mathbb{C}^{d}\right) \times \mathbb{C}^{|\mathcal{U}|} \otimes \mathbb{C}^{|\mathcal{A}|} \otimes \mathcal{L}\left(\mathbb{C}^{d}\right) \rightarrow \mathcal{L}\left(\mathbb{C}^{d}\right) \otimes \mathcal{L}\left(\mathbb{C}^{d}\right)
$$

by defining $\langle A, B\rangle_{\mu_{L}}$, for $|A\rangle=\sum_{u, a}|u\rangle|a\rangle \otimes A_{u}^{a}$ and $|B\rangle=\sum_{u, a}|u\rangle|a\rangle \otimes B_{u}^{a}$, as

$$
\langle A, B\rangle_{\mu_{L}}:=\sum_{u} \mu_{L}(u) \sum_{a} \overline{A_{u}^{a}} \otimes B_{u}^{a} .
$$

With this definition the success probability of the strategy $(A, B)$ in $G$ can be expressed as

$$
\begin{aligned}
\operatorname{VAL}^{*}(G,|A\rangle,|B\rangle) & :=\left\|\langle A,(G \otimes \mathrm{Id}) B\rangle_{\mu}\right\|_{\infty} \\
& =\left\|\sum_{u, a} \mu(u) \overline{A_{u}^{a}} \otimes\left(\sum_{v} \mu(v \mid u) \sum_{b \rightarrow a} B_{v}^{b}\right)\right\|_{\infty} \\
& =\left\|\sum_{u, v} \mu(u, v) \sum_{a, b \rightarrow a} \overline{A_{u}^{a}} \otimes B_{v}^{b}\right\|_{\infty} \\
& =\max _{|\Psi\rangle \in \mathbb{C}^{d} \otimes \mathbb{C}^{d}, \||\Psi\rangle \|=1} \sum_{u, v} \mu(u, v) \sum_{a, b \rightarrow a}\left\langle\Psi\left|\overline{A_{u}^{a}} \otimes B_{v}^{b}\right| \Psi\right\rangle .
\end{aligned}
$$

We also define the entangled value of the game, $\operatorname{VAL}^{*}(G)$, to be the highest value achievable by any quantum strategy:

$$
\begin{aligned}
\operatorname{VAL}^{*}(G) & =\sup _{|A\rangle,|B\rangle} \operatorname{VAL}^{*}(G,|A\rangle,|B\rangle) \\
& =\sup _{|A\rangle,|B\rangle}\left\|\langle A,(G \otimes \mathrm{Id}) B\rangle_{\mu}\right\|_{\infty} \\
& =\sup _{\left\{A_{u}^{a}\right\},\left\{B_{v}^{b}\right\},|\Psi\rangle} \sum_{u, v} \mu(u, v) \sum_{a, b \rightarrow a}\left\langle\Psi\left|\overline{A_{u}^{a}} \otimes B_{v}^{b}\right| \Psi\right\rangle \\
& =\sup _{\left\{A_{u}^{a}\right\},\left\{B_{v}^{b}\right\},|\Psi\rangle} \sum_{u} \mu(u) \sum_{a}\left\langle\Psi\left|\overline{A_{u}^{a}} \otimes B_{u}^{a}\right| \Psi\right\rangle,
\end{aligned}
$$

\footnotetext{
${ }^{2}$ The state $|\Psi\rangle$ is often considered to be an integral part of the strategy. However it will be more convenient for us to not fix it a priori. Given measurement operators for both players in a game, it is always clear what is the optimal choice of entangled state; it is obtained as the largest eigenvector of a given operator depending on the game and the measurements (see below).

${ }^{3}$ The complex conjugate on $A$ is not necessary, but for our purposes it is natural to include it in light of the proof of Lemma4
} 
where here we slightly abuse notation and denote

$$
B_{u}^{a}:=\left(\langle u|\langle a| \otimes \mathrm{Id})(G \otimes \mathrm{Id}) \mid B\rangle=\sum_{v} \mu(v \mid u) \sum_{b \rightarrow a} B_{v}^{b}\right.
$$

We note that in the above the supremum may in general not be attained as optimal strategies may require infinite dimensions. In this paper we always restrict ourselves to finite dimensional strategies.

We end this section with the following well-known claim which shows that in some (though not all) respects projection games are as general as arbitrary games. (See Appendix A for the proof.)

Claim 3. There exists a polynomial-time computable transformation mapping any two-player one-round game $G$ to a projection game $G^{\prime}$ such that the following hold:

$$
1-2\left(1-\operatorname{VAL}\left(G^{\prime}\right)\right) \leq \operatorname{VAL}(G) \leq \operatorname{VAL}\left(G^{\prime}\right) .
$$

In particular, $\operatorname{vAL}\left(G^{\prime}\right)=1$ if and only if $\operatorname{VAL}(G)=1$, and $1-\operatorname{VAL}\left(G^{\prime}\right)=\Theta(1-\operatorname{vaL}(G))$. Moreover, for the entangled value we have the weaker bound

$$
\operatorname{VAL}^{*}\left(G^{\prime}\right) \leq \sqrt{\frac{1+\operatorname{VAL}^{*}(G)}{2}}
$$

which implies $1-\operatorname{VAL}^{*}\left(G^{\prime}\right)=\Omega\left(1-\operatorname{VAL}^{*}(G)\right)$.

\section{Relaxations of the game value}

In this section we introduce two relaxations of the entangled value $\operatorname{VAL}^{*}(G)$ of a projection game $G$. Both are quantum analogues of relaxations in [DS13], and are used in the same way. The first relaxation, denoted

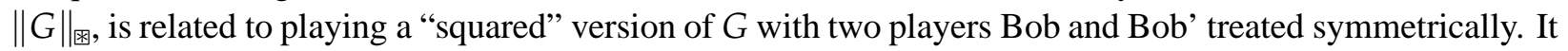
is defined in Section 3.1, and is easily seen to give a good approximation to $\mathrm{VAL}^{*}$, as shown in the following lemma (see Section 3.1 for the proof):

Lemma 4. For any projection game G,

$$
\operatorname{VAL}^{*}(G)^{2} \leq\|G\|_{\circledast 2}^{2} \leq \operatorname{VAL}^{*}(G) .
$$

The second relaxation, denoted $\operatorname{VAL}_{+}^{*}(G)$, is defined in Section 3.2 It will be proven to be a good approximation to $\|G\|_{\circledast}$ and thus to $\mathrm{VAL}^{*}$, although this will require more work.

Lemma 5. For any projection game G,

$$
\|G\|_{\circledast}^{2} \leq \operatorname{VAL}_{+}^{*}(G) \leq \varphi\left(\|G\|_{\circledast}^{2}\right),
$$

for $\varphi(x)=1-C(1-x)^{c}$ and some positive constants $C, c>0$.

The proof of Lemma 5 is given in Section 4 The definition of $\mathrm{VAL}_{+}^{*}$ is motivated by the following multiplicative property.

Lemma 6. For any two projection games $G$ and $H$,

$$
\|G \otimes H\|_{\circledast}^{2} \leq \operatorname{VAL}_{+}^{*}(G) \cdot\|H\|_{\circledast 2}^{2} .
$$


The proof of Lemma 6 is given in Section 3.2

With these three inequalities in hand we easily derive the parallel repetition theorem, Theorem 11, as follows. By repeated applications of (8), we get

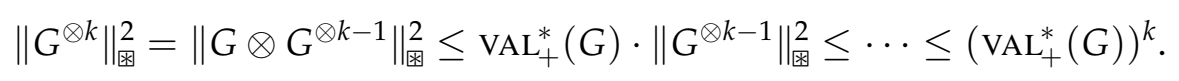

Combining with (6) and (7) we get

$$
\operatorname{VAL}^{*}\left(G^{\otimes k}\right)^{2} \leq\left\|G^{\otimes k}\right\|_{\circledast 2}^{2} \leq\left(\operatorname{VAL}_{+}^{*}(G)\right)^{k} \leq\left(\varphi\left(\|G\|_{\circledast}^{2}\right)\right)^{k} \leq\left(\varphi\left(\operatorname{VAL}^{*}(G)\right)\right)^{k},
$$

where the last step follows from (6) and the monotonicity of $\varphi$.

\subsection{The square norm}

Definition 7. For a game $G$ and a quantum strategy $|B\rangle$ write $\| G \otimes \operatorname{Id}|B\rangle \|_{\circledast}:=(\|\langle G \otimes \operatorname{Id} B, G \otimes$ $\left.\operatorname{Id} B\rangle_{\mu} \|_{\infty}\right)^{1 / 2}$ and define

$$
\|G\|_{\circledast}:=\sup _{|B\rangle} \| G \otimes \operatorname{Id}|B\rangle \|_{\text {国 }}
$$

where the supremum is taken over all quantum strategies $|B\rangle \in \mathbb{C}^{|\mathcal{V}|} \otimes \mathbb{C}^{|\mathcal{B}|} \otimes \mathcal{L}\left(\mathbb{C}^{d}\right)$.

We note that $\|\cdot\|_{\text {困 }}$ is clearly homogeneous and non-negative. Although we will not use it, one can check that $\|\cdot\|_{\circledast}$ is also definite, and hence a norm, by setting $B_{v}^{b}=\operatorname{Id}$ for every $v$ and any $b$ such that $\left(G^{\dagger} G\right)_{(v, b),(v, b)} \neq 0$ (when it exists, and for an arbitrary $b$ otherwise).

Lemma 4 claims that $\| G \otimes \operatorname{Id}|B\rangle \|_{\text {国 }}$ gives a good approximation to the maximum success probability in the game, when Bob uses the strategy specified by $|B\rangle$. We give a self-contained proof of the lemma below, but before proceeding readers familiar with quantum information theory may find it interesting to note that a direct proof of (9) can be derived using known properties of the pretty-good measurement (PGM) [HW94, $\mathrm{HJS}^{+}$96]. We briefly indicate how, before proceeding to give a self-contained proof. Suppose the second player's strategy in $G$ is fixed to $|B\rangle$. Upon receiving her question $u$, the first player has to decide on an answer $a$. She knows that the second player will receive a $v$ distributed according to $\mu(\cdot \mid u)$ and apply his measurement, obtaining an outcome $b$ and resulting in the post-measurement state $\operatorname{Tr}_{2}\left(\operatorname{Id} \otimes \sqrt{B_{v}^{b}}|\Psi\rangle\langle\Psi| \operatorname{Id} \otimes \sqrt{B_{v}^{b}}\right)$ on her system. From her point of view, she needs to provide an answer $a$ such that $\pi_{u v}(b)=a$. Only knowing $u$, her task thus amounts to optimally distinguishing between the collection of states

$$
\rho_{u}^{a}=\underset{v \sim u}{\mathrm{E}} \sum_{b \rightarrow a} \operatorname{Tr}_{2}\left(\mathrm{Id} \otimes \sqrt{B_{v}^{b}}|\Psi\rangle\langle\Psi| \operatorname{Id} \otimes \sqrt{B_{v}^{b}}\right) .
$$

If, instead of applying the optimal distinguishing measurement, Alice applied the pretty-good measurement derived from this family of states then it follows from [BK02] that the players' success probability would be at most quadratically away from what it would be were Alice to apply the optimal measurement. Using the explicit formula for the PGM one can verify that the resulting value exactly corresponds to $\| G \otimes \operatorname{Id}|B\rangle \|_{\circledast}^{2}$, which proves the first inequality in (6).

Proof of Lemma 4 We prove the following inequality, from which (6) follows by taking the supremum over all $|B\rangle$ :

$$
\max _{|A\rangle} \operatorname{VAL}^{*}(G,|A\rangle,|B\rangle)^{2} \leq \| G \otimes \operatorname{Id}|B\rangle \|_{\circledast 2}^{2} \leq \max _{|A\rangle} \operatorname{VAL}^{*}(G,|A\rangle,|B\rangle) .
$$


For the second inequality, using that $G$ is a projection game we note that for any $d$-dimensional strategy $|B\rangle$ for the second player, $(G \otimes I d)|B\rangle$ is a valid strategy for the first player, hence

$$
\|(G \otimes \operatorname{Id})|B\rangle\left\|_{\circledast \otimes}^{2}=\right\|\langle G \otimes \operatorname{Id} B, G \otimes \operatorname{Id} B\rangle_{\mu}\left\|_{\infty} \leq \max _{|A\rangle}\right\|\langle A,(G \otimes \operatorname{Id}) B\rangle_{\mu} \|_{\infty}=\max _{|A\rangle} \operatorname{VAL}^{*}(G,|A\rangle,|B\rangle) .
$$

To show the first, we write the following:

$$
\begin{aligned}
\operatorname{VAL}^{*}(G,|A\rangle,|B\rangle) & =\left\|\langle A,(G \otimes \operatorname{Id}) B\rangle_{\mu}\right\|_{\infty} \\
& =\left\|\sum_{u} \mu(u) \sum_{a} \overline{A_{u}^{a}} \otimes B_{u}^{a}\right\|_{\infty} \\
& \leq\left\|\sum_{u} \mu(u) \sum_{a} \overline{A_{u}^{a}} \otimes A_{u}^{a}\right\|_{\infty}^{1 / 2}\left\|\sum_{u} \mu(u) \sum_{a} \overline{B_{u}^{a}} \otimes B_{u}^{a}\right\|_{\infty}^{1 / 2} \\
& \leq \||(G \otimes \mathrm{Id}) B\rangle \|_{\text {国 }}
\end{aligned}
$$

where for the first inequality we used the following matrix version of the Cauchy-Schwarz inequality (see Claim 20 in Appendix $\mathrm{A}$ for a proof):

$$
\left\|\sum_{i} \overline{A_{i}} \otimes B_{i}\right\|_{\infty}^{2} \leq\left\|\sum_{i} \overline{A_{i}} \otimes A_{i}\right\|_{\infty}\left\|\sum_{i} \overline{B_{i}} \otimes B_{i}\right\|_{\infty},
$$

and the last inequality follows using $\sum_{a} A_{u}^{a} \leq \mathrm{Id}$ for every $u$, which implies

$$
\left\|\sum_{a} \overline{A_{u}^{a}} \otimes A_{u}^{a}\right\|_{\infty} \leq\left\|\sum_{a} \operatorname{Id} \otimes A_{u}^{a}\right\|_{\infty} \leq\|\operatorname{Id} \otimes \operatorname{Id}\|_{\infty}=1
$$

\subsection{The relaxation $\operatorname{VAL}_{+}^{*}(G)$}

In order to motivate our definition of $\mathrm{VAL}_{+}^{*}$, let us consider two projection games $G, H$ and any quantum strategy $|B\rangle$ for $G \otimes H$ that achieves the optimal value $\|G \otimes H\|_{\circledast}^{2}$ in the square game. Letting $\kappa:=$

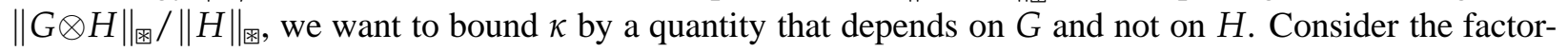
ization $G \otimes H=(G \otimes I)(I \otimes H)$ where $I$ is the identity operator; note that $I$ can also be understood as a game in which the two players are asked the same question and win if and only if they return the same answer. The application of $G \otimes H$ thus gives rise to a two step process

$$
\left|A^{\prime}\right\rangle \stackrel{G \otimes I}{\longleftarrow}|A\rangle \stackrel{I \otimes H}{\longleftarrow}|B\rangle,
$$

mapping $|B\rangle$ to $|A\rangle:=(I \otimes H \otimes \mathrm{Id})|B\rangle$ and then mapping $|A\rangle$ to $\left|A^{\prime}\right\rangle:=(G \otimes I \otimes \mathrm{Id})|A\rangle$. Let us view $|B\rangle$ as a table with rows indexed by $\mathcal{V}_{G} \times \mathcal{B}_{G}$ and columns indexed by $\mathcal{V}_{H} \times \mathcal{B}_{H}$, where $\mathcal{V}_{G}, \mathcal{V}_{H}$ and $\mathcal{B}_{G}, \mathcal{B}_{H}$ are the question and answer sets associated with the second player in $G$ and $H$ respectively, and whose entries are measurement operators, i.e. elements in $\mathcal{L}\left(\mathbb{C}^{d}\right)$. Then $|A\rangle$ is the result of applying $H \otimes \mathrm{Id}$ on each row of $|B\rangle$ separately, and we apply $G \otimes I d$ on each column of $|A\rangle$ separately to get $\left|A^{\prime}\right\rangle=(G \otimes I \otimes I d)|A\rangle$.

It is instructive to view the strategy $|B\rangle$ as an assignment to each $v \in \mathcal{V}_{G}$ and $b \in \mathcal{B}_{G}$ of a row vector $\left(\langle v|\langle b| \otimes I \otimes \mathrm{Id}) \mid B\rangle\right.$ of dimensions $\left|\mathcal{V}_{H}\right|\left|\mathcal{B}_{H}\right|$ (whose entries are again in $\mathcal{L}\left(\mathbb{C}^{d}\right)$ ). Observe that for any $v$, $\left|B_{v}\right\rangle=\sum_{b}\left(\langle v|\langle b| \otimes I \otimes I d) \mid B\rangle\right.$ is a quantum strategy for $H$, since for each question $v^{\prime}$ for $H$, the sum over answers $b^{\prime}$ of

$$
\left(\left\langle v^{\prime}\left|\left\langle b^{\prime}\right| \otimes \mathrm{Id}\right) \mid B_{v}\right\rangle=B_{v, v^{\prime}}^{b^{\prime}}=\sum_{b} B_{v, v^{\prime}}^{b, b^{\prime}}\right.
$$




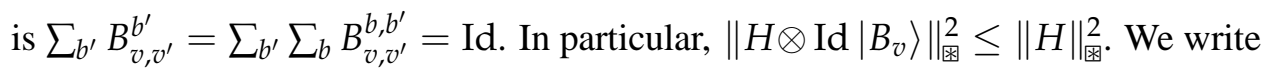

$$
\left|A_{v}\right\rangle:=\sum_{b}(\langle v|\langle b| \otimes I \otimes \mathrm{Id}) \mid A\rangle
$$

and observe that it is equal to $H \otimes \operatorname{Id}\left|B_{v}\right\rangle$, hence it satisfies $\|\left|A_{v}\right\rangle\left\|_{\circledast} \leq\right\| H \|_{\circledast}$ for every $v$. Thus the

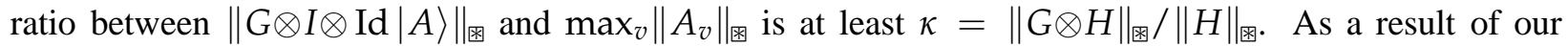
observations the ratio $\kappa$ can be upper bounded in a manner that depends only on $G$ and is independent of $H$. Abstracting the set $\mathcal{U}_{H} \times \mathcal{A}_{H}$ associated with pairs of questions and answers for the first player in $H$ as $\Omega$ for some discrete set $\Omega .4$ we are led to the definition of $\operatorname{VAL}_{+}^{*}(G)$ as the supremum of $\| G \otimes I_{\Omega} \otimes \operatorname{Id}_{\mathbb{C}^{d}}|A\rangle \|_{\text {国 }}^{2}$ ranging over vector quantum strategies $|A\rangle$ with norm $\|A\|_{+} \leq 1$ to be defined below.

Definition 8 (The relaxation $\mathrm{VAL}_{+}^{*}$ ). Let $\mathrm{G}$ be a projection game. Then

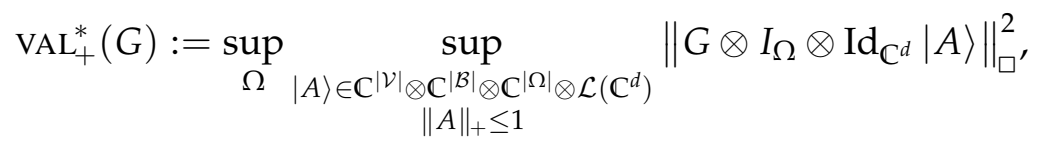

where the supremum is taken over all discrete measured spaces $\Omega$.

The definition of $\|A\|_{+}$is given by,

Definition 9 (Fractional Strategy and Vector Strategy). Let $G$ be a projection game and $\Omega$ a discrete measured space. An element

$$
|A\rangle=\sum_{v, b}|v\rangle|b\rangle \otimes A_{v}^{b} \in \mathbb{C}^{|\mathcal{V}|} \otimes \mathbb{C}^{|\mathcal{B}|} \otimes \mathcal{L}\left(\mathbb{C}^{d}\right)
$$

is a fractional quantum strategy for $G$ if for every $v, b$ the matrix $A_{v}^{b}$ is positive semidefinite and $A_{v}:=$ $\sum_{b} A_{v}^{b} \leq \mathrm{Id}$ for every $v$. A vector quantum strategy is an element

$$
|A\rangle=\sum_{\omega \in \Omega}|\omega\rangle\left|A_{\omega}\right\rangle \in \mathbb{C}^{|\Omega|} \otimes \mathbb{C}^{|\mathcal{V}|} \otimes \mathbb{C}^{|\mathcal{B}|} \otimes \mathcal{L}\left(\mathbb{C}^{d}\right)
$$

such that each $\left|A_{\omega}\right\rangle$ is a fractional quantum strategy. The norm of a vector quantum strategy is defined as

$$
\||A\rangle \|_{+}:=\left(\max _{v}\left\|\underset{\omega}{\mathrm{E}} A_{\omega v} \otimes A_{\omega v}\right\|_{\infty}\right)^{1 / 2} .
$$

With these definitions in place we prove Lemma 6 relating the square norm of a product of games to $\mathrm{VAL}_{+}^{*}$.

Proof of Lemma 6 Let $|B\rangle$ be a strategy in the square game associated to $G \otimes H$. It follows immediately from our observations above that $|A\rangle=I \otimes H \otimes I d|B\rangle$ is a vector quantum strategy for $G$ (where the space $\Omega=\mathcal{U}_{H} \times \mathcal{A}_{H}$, and the measure is the cartesian product of the probability measure $\mu_{L}$ on $\mathcal{U}_{H}$ and the counting measure on $\mathcal{A}_{H}$ ) whose norm is $\||A\rangle\left\|_{+} \leq\right\| H \|_{\text {国. This means that }}$

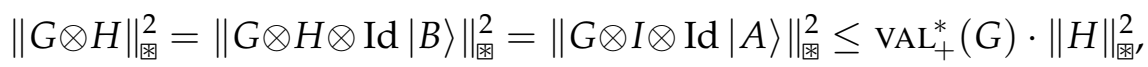

where the last inequality comes by observing that $\frac{1}{\|H\|_{\text {⿴囗十 }}}|A\rangle$ is a vector strategy with norm $\|\cdot\|_{+}$at most 1 , so its value is at most $\operatorname{VAL}_{+}^{*}(G)$. This proves the claim.

\footnotetext{
${ }^{4}$ In order for the extended inner product $\langle\cdot, \cdot\rangle_{\mu}$ to remain well-defined, we also need to equip $\Omega$ with a measure - here, it would be the cartesian product of the probability measure $\mu_{L}$ on $\mathcal{U}_{H}$ and the counting measure on $\mathcal{A}_{H}$.
} 


\section{Relating $\operatorname{VAL}_{+}^{*}(G)$ to the square norm}

In this section we prove Lemma[5, which states that $\operatorname{VAL}_{+}^{*}(G)$ is a good relaxation of the square norm $\|G\|_{\text {单 }}$ of a projection game and establishes the last step in our proof of the parallel repetition theorem, Theorem 1 We will also show that if $G$ is an expanding projection game then one can take $c=4$ in the definition of $\varphi(x)=1-C(1-x)^{c}$.

To prove the lemma, we need to show that the existence of a good vector strategy for the players in the square game $G^{\dagger} G$ implies that $\|G\|_{\text {米 }}^{2}$ is large, i.e. there also exists a good (standard) quantum strategy for the players. We will establish this by describing an explicit rounding procedure mapping the former to the latter. The rounding argument is simpler in case $G$ has the additional property of being expanding (see Section 2.2 for the definition), and we give the proof in that case in Section 4.1. In Section 4.2 we treat the case of general projection games. In that case the rounding argument is more involved and relies on a "quantum correlated sampling" lemma which is stated and proved in Section 5

In both cases, the starting point for the rounding procedure is the existence of a vector strategy $|\hat{A}\rangle$ and entangled state $|\hat{\Psi}\rangle$ satisfying inequality (13) in the following claim, which is essentially a restatement of the inequality "VAL* $(G) \geq 1-\eta$ ".

Claim 10. Let $G$ be a projection game and $\eta>0$ such that $\operatorname{VAL}_{+}^{*}(G) \geq 1-\eta$. Then there exists a discrete measured space $\Omega$, an integer $d$, a bipartite state $|\hat{\Psi}\rangle \in \mathbb{C}^{d} \otimes \mathbb{C}^{d}$ and a vector strategy $|\hat{A}\rangle \in$ $\mathbb{C}^{|\Omega|} \otimes \mathbb{C}^{|\mathcal{V}|} \otimes \mathbb{C}^{|\mathcal{B}|} \otimes \mathcal{L}\left(\mathbb{C}^{d}\right)$ such that for every $\omega$ and $v, b, \hat{A}_{\omega v}^{b} \geq 0$ and $\hat{A}_{\omega v}=\sum_{b} \hat{A}_{\omega v}^{b} \leq \mathrm{Id}$, and

$$
\underset{\omega}{\mathrm{E}} \underset{v \sim v^{\prime}}{\mathrm{E}} \sum_{b \leftrightarrow b^{\prime}}\left\langle\hat{\Psi}\left|\overline{\hat{A}}_{\omega v}^{b} \otimes \hat{A}_{\omega v^{\prime}}^{b^{\prime}}\right| \hat{\Psi}\right\rangle \geq(1-\eta) \max _{v}\left\{\underset{\omega}{\mathrm{E}}\left\langle\hat{\Psi}\left|\overline{\hat{A}_{\omega v}} \otimes \hat{A}_{\omega v}\right| \hat{\Psi}\right\rangle\right\}
$$

where formally $\mathrm{E}_{v \sim v^{\prime}} \sum_{b \leftrightarrow b^{\prime}}$ is shorthand for $\sum_{u} \mu(u) \sum_{a} \sum_{v, v^{\prime}} \mu(v \mid u) \mu\left(v^{\prime} \mid u\right) \sum_{b \rightarrow a, b^{\prime} \rightarrow a}$.

Proof. By definition of $\mathrm{VAL}_{+}^{*}$, there exists a discrete measured space $\Omega$ and a vector strategy $|\hat{A}\rangle$ such that $\||\hat{A}\rangle \|_{+}=1$ and $\| \operatorname{Id}_{\Omega} \otimes G \otimes \operatorname{Id}|\hat{A}\rangle \|_{\circledast}^{2} \geq 1-\eta$. Recalling the definition of $\|\cdot\|_{+}$(see Definition9) and of $\|\cdot\|_{\text {ख }}$ (see Definition 7), we may reformulate this statement as the inequality

$$
\left\|\underset{\omega}{\mathrm{E}} \underset{v \sim v^{\prime}}{\mathrm{E}} \sum_{b \leftrightarrow b^{\prime}} \overline{\hat{A}_{\omega v}^{b}} \otimes \hat{A}_{\omega v^{\prime}}^{b^{\prime}}\right\|_{\infty} \geq(1-\eta) \max _{v}\left\|\underset{\omega}{\mathrm{E}} \overline{\hat{A}_{\omega v}} \otimes \hat{A}_{\omega v}\right\|_{\infty} .
$$

Letting $|\hat{\Psi}\rangle$ be a state which optimizes the left-hand side gives (13).

In the following sections we show how any vector strategy $|\hat{A}\rangle$ and state $|\hat{\Psi}\rangle$ such that (13) holds can be rounded to a good strategy for the square game $G^{\dagger} G$, first in case the game $G$ is expanding and then in the case of an arbitrary projection game.

\subsection{The expanding case}

Let $|\hat{A}\rangle$ be a vector strategy and $|\hat{\Psi}\rangle$ a state such that (13) holds, and assume that $G^{\dagger} G$ is expanding. Our goal is to identify a quantum strategy $|\tilde{A}\rangle$ such that $\| G \otimes \operatorname{Id}|\tilde{A}\rangle \|_{\circledast 2}^{2} \geq 1-O\left(\eta^{1 / c}\right)$, which by Claim[10 will suffice to prove Lemma 5 for the case of expanding projection games.

Our first step consists in fixing a "good" value $\omega \in \Omega$ and restricting our attention to the fractional strategy $\left|\hat{A}_{\omega}\right\rangle:=(\langle\omega| \otimes I \otimes I d)|\hat{A}\rangle$ specified by the operators $\hat{A}_{\omega v}^{b}$ obtained from that $\omega$. Using that the max is larger than the average, Eq. (13) implies

$$
\underset{\omega}{\mathrm{E}}\left(\underset{v \sim v^{\prime}}{\mathrm{E}} \sum_{b \leftrightarrow b^{\prime}}\left\langle\hat{\Psi}\left|\overline{\hat{A}_{\omega v}^{b}} \otimes \hat{A}_{\omega v^{\prime}}^{b^{\prime}}\right| \hat{\Psi}\right\rangle\right) \geq(1-\eta) \underset{\omega}{\mathrm{E}}\left(\underset{v}{\mathrm{E}}\left\langle\hat{\Psi}\left|\overline{\hat{A}_{\omega v}} \otimes \hat{A}_{\omega v}\right| \hat{\Psi}\right\rangle\right)
$$


For the remainder of this section fix an $\omega$ such that (14) holds for that $\omega$. The only property we will need of the $\left\{\hat{A}_{\omega v}^{b}\right\}$ in order to construct a good strategy in $G^{\dagger} G$ is that they are positive semidefinite operators which satisfy that inequality. (In contrast, for the non-expanding case, Eq. (14) by itself turns out to be too weak an inequality, and we must work with 113 .)

The definition of $|\tilde{A}\rangle$ almost imposes itself: the only "defect" of $\left|\hat{A}_{\omega}\right\rangle$ is that it is only a fractional strategy, meaning that for any question $v$ the sum $\hat{A}_{\omega v}=\sum_{b} \hat{A}_{\omega v}^{b}$ may not equal the identity, but due to the "conditioning" on $\omega$ could in general be much smaller. It is natural to define a re-normalized strategy as follows. First, for every pair of questions $v, v^{\prime} \in \mathcal{V}$ and a choice of unitaries $U_{v}, U_{v^{\prime}}$ to be made later we introduce the post-measurement state

$$
\left|\Psi_{v v^{\prime}}\right\rangle:=\bar{U}_{v} \overline{\hat{A}}_{\omega v}^{1 / 2} \otimes U_{v^{\prime}} \hat{A}_{\omega v^{\prime}}^{1 / 2}|\hat{\Psi}\rangle
$$

The state $\left|\Psi_{v v^{\prime}}\right\rangle$ is the post-measurement state that corresponds to "pre-conditioning" $|\hat{\Psi}\rangle$ by applying the binary measurements $\left\{\overline{\hat{A}} \omega v, \mathrm{Id}-\overline{\hat{A}}_{\omega v}\right\}$ for the first player, $\left\{\hat{A}_{\omega v^{\prime}}, \mathrm{Id}-\hat{A}_{\omega v^{\prime}}\right\}$ for the second, and conditioning on both of them obtaining the first outcome. In general the post-measurement state is only defined up to a local unitary, and this freedom is represented in the unitaries $U_{v}$ and $U_{v^{\prime}}$. Next for every question $v \in \mathcal{V}$ and answer $b \in \mathcal{B}$ we define the measurement operator

$$
\tilde{A}_{v}^{b}:=U_{v} \hat{A}_{\omega v}^{-1 / 2} \hat{A}_{\omega v}^{b} \hat{A}_{\omega v}^{-1 / 2} U_{v}^{\dagger}
$$

where here $\hat{A}_{\omega v}^{-1 / 2}$ denotes the square root of the pseudo-inverse of $\hat{A}_{\omega v}=\sum_{b} \hat{A}_{\omega v}^{b}$. Again, there is always a unitary degree of freedom in the choice of the square root, and the unitaries $U_{v}$, the same as in (15), represent that degree of freedom. With this definition it is easy to verify that each $\tilde{A}_{v}^{b}$ is positive semidefinite and that $\sum_{b} \tilde{A}_{v}^{b} \leq \mathrm{Id}$; since we may always add a "dummy" outcome in order for the measurement operators to sum to identity, $\left\{\tilde{A}_{v}^{b}\right\}_{b}$ is a well-defined measurement and $|\tilde{A}\rangle:=\sum_{v, b}|v, b\rangle \otimes \tilde{A}_{v}^{b}$ a valid quantum strategy in $G^{\dagger} G$.

Now suppose that, upon receiving their respective questions $v$ and $v^{\prime}$, players in $G^{\dagger} G$ were to measure their respective share of the (re-normalized) state $\left|\Psi_{v v^{\prime}}\right\rangle$ using the measurements given by the $\left\{\overline{\tilde{A}_{v}^{b}}\right\}_{b}$, $\left\{\tilde{A}_{v^{\prime}}^{b^{\prime}}\right\}_{b^{\prime}}$ respectively. The probability that they obtain the pair of outcomes $\left(b, b^{\prime}\right)$ is given, up to normalization by $\|\left|\Psi_{v v^{\prime}}\right\rangle \|^{-2}$, by

$$
\begin{aligned}
\left\langle\Psi_{v v^{\prime}}\left|\tilde{A}_{v}^{b} \otimes \tilde{A}_{v^{\prime}}^{b^{\prime}}\right| \Psi_{v v^{\prime}}\right\rangle & =\langle\hat{\Psi}|\left({\overline{\hat{A}_{\omega v}}}^{1 / 2} \bar{U}_{v}^{\dagger} \otimes \hat{A}_{\omega v^{\prime}}^{1 / 2} U_{v^{\prime}}^{\dagger}\right)\left(\overline{U_{v} \hat{A}_{\omega v}^{-1 / 2} \hat{A}_{\omega v}^{b} \hat{A}_{\omega v}^{-1 / 2} U_{v}^{+}}\right. \\
& \left.\otimes U_{v^{\prime}} \hat{A}_{\omega v^{\prime}}^{-1 / 2} \hat{A}_{\omega v^{\prime}}^{b} \hat{A}_{\omega v^{\prime}}^{-1 / 2} U_{v^{\prime}}^{\dagger}\right)\left({\overline{U_{v}}}_{\hat{A}_{\omega v}}^{1 / 2} \otimes U_{v^{\prime}} \hat{A}_{\omega v^{\prime}}^{1 / 2}\right)|\hat{\Psi}\rangle \\
= & \left\langle\hat{\Psi}\left|\hat{A}_{v}^{b} \otimes \hat{A}_{v^{\prime}}^{b^{\prime}}\right| \hat{\Psi}\right\rangle,
\end{aligned}
$$

perfectly reproducing the correlations induced by the fractional strategy $\left|\hat{A}_{\omega}\right\rangle$ together with $|\hat{\Psi}\rangle$. Thus if it were the case that for all $\left(v, v^{\prime}\right),\left|\Psi_{v v^{\prime}}\right\rangle=|\Psi\rangle$, a vector independent of $\left(v, v^{\prime}\right)$, then the players could use $|\Psi\rangle$ as their initial shared entangled state and perfectly emulate $\left|\hat{A}_{\omega}\right\rangle$ using the quantum strategy $|\tilde{A}\rangle$. Noticing that the term on the right-hand side of (14), for our choice of $\omega$, is exactly $\|\left|\Psi_{v v}\right\rangle \|^{2}$, the equality (17) when combined with (14) would immediately show that the quantum strategy we have just defined would achieve a value at least $1-\eta$ in $G^{\dagger} G$, implying $\|G \otimes \operatorname{Id}\|_{\text {ख }}^{2} \geq 1-\eta$ and proving Lemma 5 ,

While it may unfortunately not be the case that the $\left|\Psi_{v v^{\prime}}\right\rangle$ are independent of $\left(v, v^{\prime}\right)$, the main claim in the proof of Lemma 5 will establish that they are close, on average. Introduce the density matrix

$$
\sigma:=\frac{\mathrm{E}_{v \sim v^{\prime}}\left|\Psi_{v v^{\prime}}\right\rangle\left\langle\Psi_{v v^{\prime}}\right|}{\mathrm{E}_{v^{\prime \prime} \sim v^{\prime \prime \prime}}||\left|\Psi_{v^{\prime \prime} v^{\prime \prime \prime}}\right\rangle \|^{2}}
$$


which is simply the normalized mixture over a random pair of neighbors $v \sim v^{\prime}$ (sampled as in $G^{\dagger} G$ ) of the vectors $\left|\Psi_{v v^{\prime}}\right\rangle$. We will prove that $\sigma$ is close, on average, to any of the (re-normalized) states $\left|\Psi_{v v^{\prime}}\right\rangle$ by proceeding in two steps. First, in Lemma 12 we will show that the unitaries $U_{v}$ can be chosen in such a way that the states $\left|\Psi_{v v^{\prime \prime}}\right\rangle$ and $\left|\Psi_{v^{\prime} v^{\prime \prime}}\right\rangle$ are close to each other, for arbitrary $v^{\prime \prime}$ but on average over neighboring $v \sim v^{\prime}$. (The proof of this claim will highlight the role played by the $U_{v}$.) Second, in Claim 14 we will use our assumption that $G$ is an expanding game in order to obtain closeness for any choice of $v, v^{\prime}$ and $v^{\prime \prime}$. The result is the following:

Claim 11. Let $G$ be an expanding projection game and $|\hat{A}\rangle,|\hat{\Psi}\rangle$ a fractional strategy and state such that (14) holds. Then there exists a choice of unitaries $U_{v}$ such that, if $\left|\Psi_{v v^{\prime}}\right\rangle$ and $\sigma$ are as defined in (15) and (18) respectively, then

$$
\underset{v \sim v^{\prime}}{\mathrm{E}}\left\|\sigma-\frac{\left|\Psi_{v v^{\prime}}\right\rangle\left\langle\Psi_{v v^{\prime}}\right|}{\left.\mathrm{E}_{v^{\prime \prime} \sim v^{\prime \prime \prime}}|\|| \Psi_{v^{\prime \prime} v^{\prime \prime \prime}}\right\rangle \|^{2}}\right\|_{1}=O\left(\eta^{1 / 4}\right) .
$$

Before giving the details of the proof of Claim 11 we first show how it lets us conclude the proof of Lemma 5 for the case of expanding games.

Proof of Lemma 5 expanding case. Let $\tilde{A}_{v}^{b}$ be the measurement operators defined in (16) from $|\hat{A}\rangle$ and the unitaries $U_{v}$ as promised in Claim 11, Let $\sigma$ be the density matrix defined from the same unitaries in (18). We evaluate the value achieved by this strategy in $G^{\dagger} G$. First note that

$$
\begin{aligned}
\underset{v \sim v^{\prime}}{\mathrm{E}} \|\left|\Psi_{v v^{\prime}}\right\rangle \|^{2} & =\underset{v \sim v^{\prime}}{\mathrm{E}}\left\langle\hat{\Psi}\left|\overline{\hat{A}_{v}} \otimes \hat{A}_{v^{\prime}}\right| \hat{\Psi}\right\rangle \\
& \leq \underset{v \sim v^{\prime}}{\mathrm{E}}\left\langle\hat{\Psi}\left|\overline{\hat{A}_{v}} \otimes \hat{A}_{v}\right| \hat{\Psi}\right\rangle^{1 / 2}\left\langle\hat{\Psi}\left|\overline{\hat{A}_{v^{\prime}}} \otimes \hat{A}_{v^{\prime}}\right| \hat{\Psi}\right\rangle^{1 / 2} \\
& \leq \underset{v}{\mathrm{E}} \|\left|\Psi_{v v}\right\rangle \|^{2},
\end{aligned}
$$

where the second line follows from Claim 20 and the third uses $2 a b \leq a^{2}+b^{2}$. We can then evaluate

$$
\begin{aligned}
\underset{v \sim v^{\prime}}{\mathrm{E}} \sum_{b \leftrightarrow b^{\prime}} \operatorname{Tr}\left(\overline{\tilde{A}_{v}^{b}} \otimes \tilde{A}_{v^{\prime}}^{b^{\prime}} \sigma\right) & \geq \underset{v \sim v^{\prime}}{\mathrm{E}} \sum_{b \leftrightarrow b^{\prime}} \frac{\left\langle\Psi_{v v^{\prime}}\left|\overline{\tilde{A}_{v}^{b}} \otimes \tilde{A}_{v^{\prime}}^{b^{\prime}}\right| \Psi_{v v^{\prime}}\right\rangle}{\mathrm{E}_{v^{\prime \prime} \sim v^{\prime \prime \prime}}||\left|\Psi_{v^{\prime \prime} v^{\prime \prime \prime}}\right\rangle \|^{2}}-\underset{v \sim v^{\prime}}{\mathrm{E}}\left\|\sigma-\frac{\left|\Psi_{v v^{\prime}}\right\rangle\left\langle\Psi_{v v^{\prime}}\right|}{\mathrm{E}_{v^{\prime \prime} \sim v^{\prime \prime \prime}}||\left|\Psi_{v^{\prime \prime} v^{\prime \prime \prime}}\right\rangle \|^{2}}\right\|_{1} \\
& \geq \underset{v \sim v^{\prime}}{\mathrm{E}} \sum_{b \leftrightarrow b^{\prime}} \frac{\left\langle\Psi_{v v^{\prime}}\left|\overline{\tilde{A}_{v}^{b}} \otimes \tilde{A}_{v^{\prime}}^{b^{\prime}}\right| \Psi_{v v^{\prime}}\right\rangle}{\mathrm{E}_{v^{\prime \prime}} \|\left|\Psi_{v^{\prime \prime} v^{\prime \prime}}\right\rangle \|^{2}}-O\left(\eta^{1 / 4}\right) \\
& =\underset{v \sim v^{\prime}}{\mathrm{E}} \sum_{b \leftrightarrow b^{\prime}} \frac{\left\langle\hat{\Psi}\left|\overline{\hat{A}_{v}^{b}} \otimes \hat{A}_{v^{\prime}}^{b^{\prime}}\right| \Psi\right\rangle}{\mathrm{E}_{v^{\prime \prime}} \|\left|\Psi_{v^{\prime \prime} v^{\prime \prime}}\right\rangle \|^{2}}-O\left(\eta^{1 / 4}\right) \\
& \geq 1-\eta-O\left(\eta^{1 / 4}\right),
\end{aligned}
$$

where the first inequality uses that $|\tilde{A}\rangle$ is a strategy to bound $\mathrm{E}_{v \sim v^{\prime}} \sum_{b \leftrightarrow b^{\prime}} \overline{\tilde{A}_{v}^{b}} \otimes \tilde{A}_{v^{\prime}}^{b^{\prime}} \leq \mathrm{Id}$, the second line uses (19) for the first term and the bound from Claim 11 for the second, the third follows from (17) and the last is (14), for the particular $\omega$ that we fixed. To conclude, note that by definition

$$
\begin{aligned}
\| G \otimes \operatorname{Id}|\tilde{A}\rangle \|_{\circledast}^{2} & =\sup _{|\Psi\rangle} \underset{v \sim v^{\prime}}{\mathrm{E}} \sum_{b \leftrightarrow b^{\prime}}\left\langle\Psi\left|\overline{\tilde{A}_{v}^{b}} \otimes \tilde{A}_{v^{\prime}}^{b^{\prime}}\right| \Psi\right\rangle \\
& \geq \underset{v \sim v^{\prime}}{\mathrm{E}} \sum_{b \leftrightarrow b^{\prime}} \operatorname{Tr}\left(\left(\overline{\tilde{A}_{v}^{b}} \otimes \tilde{A}_{v^{\prime}}^{b^{\prime}}\right) \sigma\right),
\end{aligned}
$$

which is $1-O\left(\eta^{1 / 4}\right)$ by (20). 
In order for the proof of Lemma 5 to be complete it only remains to show Claim 11 As outlined above, we do this in two steps. The first step is stated as the following general lemma, which does not use the expansion condition (we will re-use the lemma in the proof of Lemma 5 for the non-expanding case treated in the next section).

Lemma 12. Let $|\Phi\rangle$ be a permutation-invariant state, $0 \leq A_{v} \leq \mathrm{Id}$, and $v$ a distribution on $\mathcal{V} \times \mathcal{V}$ that is symmetric under permutation of the two coordinates, such that

$$
\underset{v \sim v^{\prime}}{\mathrm{E}}\left\langle\Phi\left|\overline{A_{v}} \otimes A_{v^{\prime}}\right| \Phi\right\rangle \geq(1-\eta) \underset{v}{\mathrm{E}}\left\langle\Phi\left|\overline{A_{v}} \otimes A_{v}\right| \Phi\right\rangle .
$$

Then there exists unitaries $U_{v}$ such that, letting

$$
\left|\Phi_{v v^{\prime}}\right\rangle:={\overline{U_{v}}}_{A_{v}}^{1 / 2} \otimes U_{v^{\prime}} A_{v}^{1 / 2}|\Phi\rangle,
$$

we have that for any $v^{\prime \prime} \in \mathcal{V}$,

$$
\underset{v \sim v^{\prime}}{\mathrm{E}} \|\left|\Phi_{v v^{\prime \prime}}\right\rangle-\left|\Phi_{v^{\prime} v^{\prime \prime}}\right\rangle\left\|^{2}=O\left(\eta^{1 / 2}\right)\left(\underset{v}{\mathrm{E}} \|\left|\Phi_{v v}\right\rangle \|^{2}\right)^{1 / 2}\right\|\left|\Phi_{v^{\prime \prime} v^{\prime \prime}}\right\rangle \|
$$

and

$$
\underset{v \sim v^{\prime}}{\mathrm{E}} \|\left|\Phi_{v v}\right\rangle-\left|\Phi_{v^{\prime} v}\right\rangle\left\|^{2}=O\left(\eta^{1 / 2}\right) \underset{v}{\mathrm{E}}\right\|\left|\Phi_{v v}\right\rangle \|^{2} .
$$

Proof. Let $\rho$ be the reduced density of $|\Phi\rangle$ on either subsystem. Let $U_{v}$ be a unitary such that

$$
U_{v} A_{v}^{1 / 2} \rho^{1 / 4}=\rho^{1 / 4} A_{v}^{1 / 2} U_{v}^{\dagger}=\left(\rho^{1 / 4} A_{v} \rho^{1 / 4}\right)^{1 / 2}
$$

is Hermitian positive semidefinite; such a unitary can be obtained from the singular value decomposition of $A_{v}^{1 / 2} \rho^{1 / 4}$. Let $X_{v}$ be defined as

$$
X_{v}:=U_{v} A_{v}^{1 / 2} \rho^{1 / 4}=\rho^{1 / 4} A_{v}^{1 / 2} U_{v}^{\dagger} .
$$

By (22), $X_{v}$ is positive semidefinite. With this notation we have the following useful identities.

Claim 13. For every $v, v^{\prime} \in \mathcal{V}$ we have

$$
\operatorname{Tr}\left(X_{v}^{4}\right)=\operatorname{Tr}\left(\left(X_{v} X_{v}^{\dagger}\right)^{2}\right)=\left\langle\Phi\left|\overline{A_{v}} \otimes A_{v}\right| \Phi\right\rangle=\|\left|\Phi_{v v}\right\rangle \|^{2}
$$

and

$$
\operatorname{Tr}\left(X_{v}^{2} X_{v^{\prime}}^{2}\right)=\left\langle\Phi\left|\overline{A_{v}} \otimes A_{v^{\prime}}\right| \Phi\right\rangle
$$

Proof. For (24) we use the definition of $X_{v}$ to write

$$
\operatorname{Tr}\left(X_{v}^{4}\right)=\operatorname{Tr}\left(\left(X_{v} X_{v}^{\dagger}\right)^{2}\right)=\operatorname{Tr}\left(A_{v} \rho^{1 / 2} A_{v} \rho^{1 / 2}\right)=\left\langle\Phi\left|\overline{A_{v}} \otimes A_{v}\right| \Phi\right\rangle,
$$

where the last equality follows from Ando's identity, Claim 19, together with our assumption on $|\Phi\rangle$ being permutation-invariant. To show (25), expand using the definition (23)

$$
\begin{aligned}
\operatorname{Tr}\left(X_{v}^{2} X_{v^{\prime}}^{2}\right) & =\operatorname{Tr}\left(U_{v} A_{v}^{1 / 2} \rho^{1 / 2} A_{v}^{1 / 2} U_{v}^{\dagger} U_{v^{\prime}} A_{v^{\prime}}^{1 / 2} \rho^{1 / 2} A_{v^{\prime}}^{1 / 2} U_{v^{\prime}}^{\dagger}\right) \\
& =\operatorname{Tr}\left(A_{v} \rho^{1 / 2} A_{v^{\prime}} \rho^{1 / 2}\right) \\
& =\left\langle\Phi\left|\overline{A_{v}} \otimes A_{v^{\prime}}\right| \Phi\right\rangle,
\end{aligned}
$$

where the second equality follows from (22) and the last from Claim 19 
Now for any three $v, v^{\prime}, v^{\prime \prime}$,

$$
\begin{aligned}
\|\left|\Phi_{v v^{\prime \prime}}\right\rangle-\left|\Phi_{v^{\prime} v^{\prime \prime}}\right\rangle \|^{2} & =\left(\left\langle\Phi_{v v^{\prime \prime}}\left|-\left\langle\Phi_{v^{\prime} v^{\prime \prime}}\right|\right)\left(\left|\Phi_{v v^{\prime \prime}}\right\rangle-\left|\Phi_{v^{\prime} v^{\prime \prime}}\right\rangle\right)\right.\right. \\
& =\left\langle\Phi\left|\left(\overline{A_{v}^{1 / 2} U_{v}^{\dagger}}-\overline{A_{v^{\prime}}^{1 / 2} U_{v^{\prime}}^{\dagger}}\right)\left(\overline{U_{v} A_{v}^{1 / 2}}-\overline{U_{v^{\prime}} A_{v^{\prime}}^{1 / 2}}\right) \otimes A_{v^{\prime \prime}}^{1 / 2} U_{v^{\prime \prime}}^{\dagger} U_{v^{\prime \prime}} A_{v^{\prime \prime}}^{1 / 2}\right| \Phi\right\rangle \\
& =\operatorname{Tr}\left(\left(X_{v}-X_{v^{\prime}}\right)^{\dagger}\left(X_{v}-X_{v^{\prime}}\right) X_{v^{\prime \prime}}^{\dagger} X_{v^{\prime \prime}}\right) \\
& \leq\left(\operatorname{Tr}\left(\left(X_{v}-X_{v^{\prime}}\right)^{4}\right)\right)^{1 / 2}\left(\operatorname{Tr}\left(X_{v^{\prime \prime}}^{4}\right)\right)^{1 / 2},
\end{aligned}
$$

where the last inequality follows from Cauchy-Schwarz and the fact that the $X_{v}$ are positive semidefinite. The first term on the right-hand side of (26) can be bounded as

$$
\begin{aligned}
\operatorname{Tr}\left(\left(X_{v}-X_{v^{\prime}}\right)^{4}\right) & \leq \operatorname{Tr}\left(\left(X_{v}^{2}-X_{v^{\prime}}^{2}\right)^{2}\right) \\
& =\left\langle\Phi\left|\overline{A_{v}} \otimes A_{v}\right| \Phi\right\rangle+\left\langle\Phi\left|\overline{A_{v^{\prime}}} \otimes A_{v^{\prime}}\right| \Phi\right\rangle-2\left\langle\Phi\left|\overline{A_{v}} \otimes A_{v^{\prime}}\right| \Phi\right\rangle,
\end{aligned}
$$

where the first inequality can be found as e.g. Corollary 2 in [Kit86] and the equality follows from (24) and (25). Going back to (26), we obtain

$$
\underset{v \sim v^{\prime}}{\mathrm{E}} \|\left|\Phi_{v v^{\prime \prime}}\right\rangle-\left|\Phi_{v^{\prime} v^{\prime \prime}}\right\rangle\left\|^{2} \leq\left(2 \eta \underset{v}{\mathrm{E}} \|\left|\Phi_{v v}\right\rangle \|^{2}\right)^{1 / 2}\left(\|\left|\Phi_{v^{\prime \prime} v^{\prime \prime}}\right\rangle \|^{2}\right)^{1 / 2}\right.
$$

where the first inequality uses the assumption made in the lemma to bound the first term in (26) and (24) to rewrite the second. This proves the first inequality claimed in the lemma. The second is obtained by taking $v^{\prime \prime}=v$ in (26), and then the expectation over $v \sim v^{\prime}$ as in the above.

In case $G$ is expanding, we can extend the bound from Lemma 12 to apply to any triple $\left(v, v^{\prime}, v^{\prime \prime}\right)$, instead of only to neighbors $v \sim v^{\prime}$.

Claim 14. Let $0 \leq A_{v} \leq \mathrm{Id}, v$ and $|\Phi\rangle$ satisfy the assumptions of Lemma 12 and in particular (21). Let $H=\sum_{v, v^{\prime}} v\left(v, v^{\prime}\right)|v\rangle\left\langle v^{\prime}\right|$ be the "adjacency matrix" associated to $v, D=\sum_{v} v(v)|v\rangle\langle v|$ (where $v(v)$ is the marginal) and $L=\mathrm{Id}-D^{-1 / 2} H D^{-1 / 2}$ the normalized Laplacian. Suppose that the second smallest eigenvalue $\lambda_{2}$ of $L$ is positive. Then for any $v^{\prime \prime}$,

$$
\underset{v, v^{\prime}}{\mathrm{E}} \|\left|\Phi_{v v^{\prime \prime}}\right\rangle-\left|\Phi_{v^{\prime} v^{\prime \prime}}\right\rangle\left\|^{2}=O\left(\eta^{1 / 2} \lambda_{2}^{-1}\right)\left(\underset{v}{\mathrm{E}} \|\left|\Phi_{v v}\right\rangle \|^{2}\right)^{1 / 2}\right\|\left|\Phi_{v^{\prime \prime} v^{\prime \prime}}\right\rangle \| .
$$

Proof. Fix a $v^{\prime \prime}$ and let $|\phi\rangle=\sum_{v} \sqrt{v(v)}|v\rangle\left|\Phi_{v v^{\prime \prime}}\right\rangle$. Then

$$
\langle\phi|L \otimes \operatorname{Id}| \phi\rangle=\frac{1}{2} \underset{v \sim v^{\prime}}{\mathrm{E}} \|\left|\Phi_{v v^{\prime \prime}}\right\rangle-\left|\Phi_{v^{\prime} v^{\prime \prime}}\right\rangle \|^{2} .
$$

Decompose $|\phi\rangle=\left|v_{1}\right\rangle\left|\phi_{1}\right\rangle+\left|v_{2}\right\rangle\left|\phi_{2}\right\rangle$, where $\left|v_{1}\right\rangle=\sum_{v} \sqrt{v(v)}|v\rangle,\left|\phi_{1}\right\rangle=\sum_{v} v(v)\left|\Phi_{v v^{\prime \prime}}\right\rangle$ and $\left|v_{2}\right\rangle$ is orthogonal to $\left|v_{1}\right\rangle$. Since $\left\langle v_{1}|L| v_{1}\right\rangle=0$ we get from (27) that

$$
\frac{1}{2} \underset{v \sim v^{\prime}}{\mathrm{E}} \|\left|\Phi_{v v^{\prime \prime}}\right\rangle-\left|\Phi_{v^{\prime} v^{\prime \prime}}\right\rangle\left\|^{2}=\left\langle v_{2}\left|\left\langle\phi_{2}|L \otimes \operatorname{Id}| v_{2}\right\rangle\right| \phi_{2}\right\rangle \geq \lambda_{2}\right\| \phi_{2} \|^{2},
$$

where $\lambda_{2}$ is the second smallest eigenvalue of $L$ (with $\lambda_{1}=0$ ). Applying the bound from Lemma 12 we get

$$
\left\|\phi_{2}\right\|^{2}=O\left(\eta^{1 / 2} \lambda_{2}^{-1}\right)\left(\underset{v}{\mathrm{E}} \|\left|\Phi_{v v}\right\rangle \|^{2}\right)^{1 / 2} \|\left|\Phi_{v^{\prime \prime} v^{\prime \prime}}\right\rangle \|
$$


hence

$$
\||\phi\rangle-\left|v_{1}\right\rangle\left|\phi_{1}\right\rangle\left\|^{2}=\underset{v}{\mathrm{E}}\right\|\left|\Phi_{v v^{\prime \prime}}\right\rangle-\underset{v^{\prime}}{\mathrm{E}}\left|\Phi_{v^{\prime} v^{\prime \prime}}\right\rangle\left\|^{2}=O\left(\eta^{1 / 2} \lambda_{2}^{-1}\right)\left(\underset{v}{\mathrm{E}} \|\left|\Phi_{v v}\right\rangle \|^{2}\right)^{1 / 2}\right\|\left|\Phi_{v^{\prime \prime} v^{\prime \prime}}\right\rangle \| .
$$

The claimed bound follows by applying the triangle inequality.

The proof of Claim 11 follows easily from Lemma 12 and Claim 14

Proof of Claim 11] Applying Claim 14, for $v$ taken as the distribution of questions in $G^{\dagger} G$ and $|\Phi\rangle, A_{v}^{b}$ as $|\hat{\Psi}\rangle, \hat{A}_{\omega v}^{b}$, we obtain that there exists unitaries such that the states $\left|\Psi_{v v^{\prime}}\right\rangle$ defined from these unitaries as in (15) satisfy that, for any $v^{\prime \prime}$,

$$
\underset{v, v^{\prime}}{\mathrm{E}} \|\left|\Psi_{v v^{\prime \prime}}\right\rangle-\left|\Psi_{v^{\prime} v^{\prime \prime}}\right\rangle\left\|^{2}=O\left(\eta^{1 / 2} \lambda_{2}^{-1}\right)\left(\underset{v}{\mathrm{E}} \|\left|\Psi_{v v}\right\rangle \|^{2}\right)^{1 / 2}\right\|\left|\Psi_{v^{\prime \prime} v^{\prime \prime}}\right\rangle \|
$$

In addition, from the second inequality in Lemma 12 we get

$$
\underset{v, v^{\prime}}{\mathrm{E}} \|\left|\Psi_{v v^{\prime}}\right\rangle-\left|\Psi_{v v}\right\rangle\left\|^{2}=O\left(\eta^{1 / 2}\right) \underset{v}{\mathrm{E}}\right\|\left|\Psi_{v v}\right\rangle \|^{2}
$$

from which it also follows that

$$
\begin{aligned}
\left.\left|\underset{v \sim v^{\prime}}{\mathrm{E}} \|\right| \Psi_{v v^{\prime}}\right\rangle\left\|^{2}-\underset{v}{\mathrm{E}}\right\|\left|\Psi_{v v}\right\rangle \|^{2} \mid & \leq \underset{v \sim v^{\prime}}{\mathrm{E}} \|\left|\Psi_{v v^{\prime}}\right\rangle-\left|\Psi_{v v}\right\rangle\left\|\left(\|\left|\Psi_{v v}\right\rangle\|+\|\left|\Psi_{v v}\right\rangle\left\|^{1 / 2}\right\|\left|\Psi_{v^{\prime} v^{\prime}}\right\rangle \|^{1 / 2}\right)\right. \\
& \leq 2\left(\underset{v \sim v^{\prime}}{\mathrm{E}} \|\left|\Psi_{v v^{\prime}}\right\rangle-\left|\Psi_{v v}\right\rangle \|^{2}\right)^{1 / 2}\left(\underset{v}{\mathrm{E}} \|\left|\Psi_{v v}\right\rangle \|^{2}\right)^{1 / 2} \\
& =O\left(\eta^{1 / 2}\right)\left(\underset{v}{\mathrm{E}} \|\left|\Psi_{v v}\right\rangle \|^{2}\right)^{1 / 2},
\end{aligned}
$$

where the first inequality uses $\left|a^{2}-b^{2}\right| \leq|a-b||a+b|$ and the Cauchy-Schwarz inequality to bound $\|\left|\Psi_{v v^{\prime}}\right\rangle\|\leq\|\left|\Psi_{v v}\right\rangle\left\|^{1 / 2}\right\|\left|\Psi_{v^{\prime} v^{\prime}}\right\rangle \|^{1 / 2}$, the second uses Cauchy-Schwarz, and the last (29).

We now evaluate the overlap

$$
\begin{aligned}
\underset{v \sim v^{\prime}}{\mathrm{E}}\left\langle\Psi_{v v^{\prime}}|\sigma| \Psi_{v v^{\prime}}\right\rangle & =\underset{v \sim v^{\prime} v^{\prime \prime} \sim v^{\prime \prime \prime}}{\mathrm{E}} \frac{\left|\left\langle\Psi_{v v^{\prime}} \mid \Psi_{v^{\prime \prime} v^{\prime \prime \prime}}\right\rangle\right|^{2}}{\underset{v \sim v^{\prime}}{\mathrm{E}} \|\left|\Psi_{v v^{\prime}}\right\rangle \|^{2}} \\
& \geq \frac{\left(\underset{v \sim v^{\prime}}{\mathrm{E}}\left\|\Psi_{v v^{\prime}}\right\|\right)^{2}-O\left(\eta^{1 / 2}\right)\left(\underset{v}{\mathrm{E}} \|\left|\Psi_{v v}\right\rangle \|^{2}\right)^{2}}{\underset{v \sim v^{\prime}}{\mathrm{E}} \|\left|\Psi_{v v^{\prime}}\right\rangle \|^{2}} \\
& \geq\left(1-O\left(\eta^{1 / 2}\right) \underset{v}{\mathrm{E}}\left\|\Psi_{v v}\right\|^{2},\right.
\end{aligned}
$$

where the second line follows from (28) and the last uses (30). Applying the Fuchs-van-de-Graaf inequality [FvdG99],

$$
\begin{aligned}
\underset{v \sim v^{\prime}}{\mathrm{E}}\left\|\sigma-\frac{\left|\Psi_{v v^{\prime}}\right\rangle\left\langle\Psi_{v v^{\prime}}\right|}{\mathrm{E}_{v^{\prime \prime} \sim v^{\prime \prime \prime}}||\left|\Psi_{v^{\prime \prime} v^{\prime \prime \prime}}\right\rangle \|^{2}}\right\|_{1} & \leq\left(1-\frac{\mathrm{E}_{v \sim v^{\prime}}\left\langle\Psi_{v v^{\prime}}|\sigma| \Psi_{v v^{\prime}}\right\rangle}{\mathrm{E}_{v^{\prime \prime} \sim v^{\prime \prime \prime}}||\left|\Psi_{v^{\prime \prime} v^{\prime \prime \prime}}\right\rangle \|^{2}}\right)^{1 / 2} \\
& =O\left(\eta^{1 / 4}\right)
\end{aligned}
$$

where the second inequality uses (30) and (31). 


\subsection{Non-expanding games}

Suppose $G$ is an arbitrary (not necessarily expanding) projection game. In the game $G^{\dagger} G$ the players are always sent neighboring $v \sim v^{\prime}$. To mimic the proof strategy from the previous section, we would like to enable the players to take advantage of the possibility of using an arbitrary entangled state in order to initialize themselves in a state that is close to $\left|\Psi_{v v^{\prime}}\right\rangle$. The difficulty is that this must be done "on the fly", as $\left|\Psi_{v v^{\prime}}\right\rangle$ depends on the questions $v, v^{\prime}$; indeed since $G$ is not expanding there is no single state close to all $\left|\Psi_{v v^{\prime}}\right\rangle$ that they could have agreed upon before the start of the game (as was the role of $\sigma$ in the previous section).

At this point it is natural to attempt to resort to the use of a so-called family of "universal embezzling states" $\left|\Gamma_{d}\right\rangle \in \mathbb{C}^{d} \otimes \mathbb{C}^{d}$. These states, introduced in [vH03], have the property that for any given state $|\psi\rangle$ there exists a $d$ and unitaries $U, V$ such that $U \otimes V\left|\Gamma_{d}\right\rangle \approx|\psi\rangle\left|\Gamma_{d^{\prime}}\right\rangle$ for some $d^{\prime}$. Hence if both players have a description of the target state $\left|\Psi_{v v^{\prime}}\right\rangle$ they can easily generate it locally from the universal state $\left|\Gamma_{d}\right\rangle$.

The difficulty, however, is that only the first player knows $v$, and the second knows $v^{\prime}$ : how to make them agree on which state to embezzle? Lemma 12 suggests a solution. Applied to the present setting, the lemma implies that

$$
\underset{v \sim v^{\prime}}{\mathrm{E}} \|\left|\Psi_{v v^{\prime}}\right\rangle-\left|\Psi_{v v}\right\rangle\left\|^{2}=O\left(\eta^{1 / 2}\right) \underset{v}{\mathrm{E}}\right\|\left|\Psi_{v v}\right\rangle \|^{2},
$$

that is, all three states $\left|\Psi_{v v^{\prime}}\right\rangle,\left|\Psi_{v v}\right\rangle$ and $\left|\Psi_{v^{\prime} v^{\prime}}\right\rangle$ are close for neighboring $v \sim v^{\prime}$. Hence the first player, knowing her question $v$, can compute a classical description of the state $\left|\Psi_{v v}\right\rangle$; the second player can compute a classical description of $\left|\Psi_{v^{\prime} v^{\prime}}\right\rangle$. These two states are close to each other as well to the target state: are these conditions sufficient for the two players to successfully embezzle a joint state close to either of the three?

It turns out that, if one naïvely applies the embezzling procedure described in [vH03] to this setting, it can fail completely even when the states are arbitrarily close (see Section 5 for an example). Nevertheless, in the next section we state and prove a "quantum correlated sampling lemma", which extends the results in [vH03] to this "approximate" scenario. Based on that lemma it is not hard to adapt the proof from the previous section, as follows.

Proof of Lemma 5 . Let $|\hat{A}\rangle$ be a vector strategy, and $|\hat{\Psi}\rangle$ a state such that (13) holds. Our goal is to identify a quantum strategy $|\tilde{A}\rangle$ such that $\| G \otimes \operatorname{Id}|\tilde{A}\rangle \|_{\circledast}^{2} \geq 1-O\left(\eta^{1 / c}\right)$, which by Claim 10 will suffice to prove Lemma5,

We define a "re-normalized" vector strategy $|\tilde{A}\rangle \in \mathbb{C}^{|\Omega|} \otimes \mathbb{C}^{|\mathcal{V}|} \otimes \mathbb{C}^{|\mathcal{B}|} \otimes \mathcal{L}\left(\mathbb{C}^{d}\right)$, from which we will later obtain a quantum strategy $\left|\tilde{A}_{\omega}\right\rangle$ by making a good choice of $\omega \in \Omega$. As in the previous section, for every $\omega$ we may define states

$$
\left|\Psi_{\omega v v^{\prime}}\right\rangle:={\overline{U_{\omega v}}}_{\hat{A}_{\omega v}}^{1 / 2} \otimes U_{\omega v^{\prime}} \hat{A}_{\omega v^{\prime}}^{1 / 2}|\hat{\Psi}\rangle,
$$

where the $U_{\omega v}$ are the unitaries given by Lemma 12, as a consequence of (13) (replacing the max on the right-hand-side by an average) the assumption of the lemma is satisfied, on average over $\omega \in \Omega$, for the states $\left|\Psi_{\omega v v^{\prime}}\right\rangle$. The lemma gives us the following bound:

$$
\underset{\omega v \sim v^{\prime}}{\mathrm{E}} \|\left|\Psi_{\omega v v}\right\rangle-\left|\Psi_{\omega v v^{\prime}}\right\rangle\left\|^{2}=O\left(\eta^{1 / 2}\right) \underset{\omega}{\mathrm{E}} \underset{v}{\mathrm{E}}\right\|\left|\Psi_{\omega v v}\right\rangle \|^{2} .
$$

In addition, for every $\omega$ and question $v \in \mathcal{V}$ let $\overline{V_{\omega v}}$ and $W_{\omega v}$ be the unitaries that are defined in Lemma15, for the (re-normalized) state $\left|\Psi_{\omega v v}\right\rangle$ and a choice of $\delta=\eta$. By convexity the lemma gives us that

$$
\underset{\omega v \sim v^{\prime}}{\mathrm{E}} \| \overline{V_{\omega v}} \otimes W_{\omega v^{\prime}}\left|\Gamma_{d d^{\prime}}\right\rangle-\|\left|\Psi_{\omega v v}\right\rangle \|^{-1}\left|\Psi_{\omega v v}\right\rangle\left|\Gamma_{d^{\prime}}\right\rangle \|^{2}=O\left(\left\|\frac{\left|\Psi_{\omega v v}\right\rangle}{\|\left|\Psi_{\omega v v}\right\rangle \|}-\frac{\left|\Psi_{\omega v^{\prime} v^{\prime}}\right\rangle}{\|\left|\Psi_{\omega v^{\prime} v^{\prime}}\right\rangle \|}\right\|^{2 / 6}\right) .
$$


For any question $v \in \mathcal{V}$ and answer $b \in \mathcal{B}$, define measurement operators

$\tilde{A}_{\omega v}^{b}:=V_{\omega v}^{+}\left(U_{\omega v} \hat{A}_{\omega v}^{-1 / 2} \hat{A}_{\omega v}^{b} \hat{A}_{\omega v}^{-1 / 2} U_{\omega v}^{+} \otimes \operatorname{Id}_{d^{\prime}}\right) V_{\omega v}, \tilde{B}_{\omega v}^{b}:=W_{\omega v}^{+}\left(U_{\omega v} \hat{A}_{\omega v}^{-1 / 2} \hat{A}_{\omega v}^{b} \hat{A}_{\omega v}^{-1 / 2} U_{\omega v}^{\dagger} \otimes \operatorname{Id}_{d^{\prime}}\right) W_{\omega v}$.

It is easy to verify that each $\tilde{A}_{\omega v}^{b}$ and $\tilde{B}_{\omega v}^{b}$ is positive semidefinite, and that $\sum_{b} \tilde{A}_{\omega v}^{b}, \sum_{b} \tilde{B}_{\omega v}^{b} \leq \mathrm{Id}$. Since we may always add a "dummy" outcome in order for the measurement operators to sum to identity, both $\left\{\tilde{A}_{\omega v}^{b}\right\}_{b}$ and $\left\{\tilde{B}_{\omega v}^{b}\right\}_{b}$ are well-defined measurements, and for every $\omega,\left|\tilde{A}_{\omega}\right\rangle:=\sum_{v, b}|v, b\rangle \otimes \tilde{A}_{\omega v}^{b}$ and $\left|\tilde{B}_{\omega}\right\rangle:=\sum_{v, b}|v, b\rangle \otimes \tilde{B}_{\omega v}^{b}$ valid strategies for the players in $G^{\dagger} G$ (we will soon show that at least one of these strategies must be a good strategy for the square game).

We can first bound

$$
\begin{aligned}
& \underset{\omega v \sim v^{\prime}}{\mathrm{E}} \| \overline{V_{\omega v}} \otimes W_{\omega v^{\prime}}\left|\Gamma_{d d^{\prime}}\right\rangle-\|\left|\Psi_{\omega v v}\right\rangle \|^{-1}\left|\Psi_{\omega v v^{\prime}}\right\rangle\left|\Gamma_{d^{\prime}}\right\rangle \|^{2} \\
& \leq \underset{\omega v \sim v^{\prime}}{\mathrm{E}} \| \overline{V_{\omega v}} \otimes W_{\omega v^{\prime}}\left|\Gamma_{d d^{\prime}}\right\rangle-\|\left|\Psi_{\omega v v}\right\rangle \|^{-1}\left|\Psi_{\omega v v}\right\rangle\left|\Gamma_{d^{\prime}}\right\rangle\left\|^{2}+\underset{\omega v \sim v^{\prime}}{\mathrm{E}}\right\|\left|\Psi_{\omega v v}\right\rangle\left\|^{-2}\right\|\left|\Psi_{\omega v v}\right\rangle-\left|\Psi_{\omega v v^{\prime}}\right\rangle \|^{2} \\
& =O\left(\underset{\omega}{\mathrm{E}} \underset{v \sim v^{\prime}}{\mathrm{E}}\left\|\frac{\left|\Psi_{\omega v v}\right\rangle}{\|\left|\Psi_{\omega v v}\right\rangle}-\frac{\left|\Psi_{\omega v^{\prime} v^{\prime}}\right\rangle}{\|\left|\Psi_{\omega v^{\prime} v^{\prime}}\right\rangle}\right\|^{2 / 6}\right)+O\left(\eta^{1 / 2}\right) \\
& =O\left(\underset{\omega v \sim v^{\prime}}{\mathrm{E}} \mathrm{E} \|\left|\Psi_{\omega v v}\right\rangle\left\|^{-1 / 6}\right\|\left|\Psi_{\omega v^{\prime} v^{\prime}}\right\rangle\left\|^{-1 / 6}\right\|\left|\Psi_{\omega v v}\right\rangle-\left|\Psi_{\omega v^{\prime} v^{\prime}}\right\rangle \|^{2 / 6}\right)+O\left(\eta^{1 / 2}\right) \\
& =O\left(\eta^{1 / 6}\right) \text {, }
\end{aligned}
$$

where in the second line we used (33) and the Cauchy-Schwarz inequality to bound the last term, and (34) for the first; in the third line we used that $\|\left|\Psi_{v v}\right\rangle \| \leq 1$, and in the last we again applied (33) and the Cauchy-Schwarz inequality. Note that

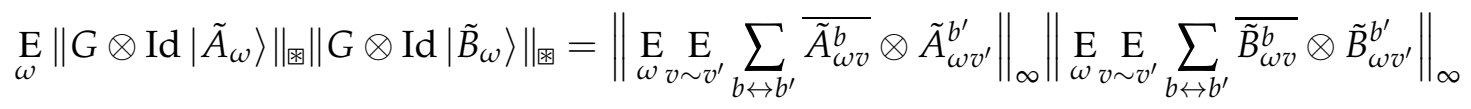

$$
\begin{aligned}
& \geq\left\|\underset{\omega v \sim v^{\prime}}{\mathrm{E}} \sum_{b \leftrightarrow b^{\prime}} \overline{\tilde{A}_{\omega v}^{b}} \otimes \tilde{B}_{\omega v^{\prime}}^{b^{\prime}}\right\|_{\infty}^{2}
\end{aligned}
$$

where the last inequality follows from Claim 20. Hence

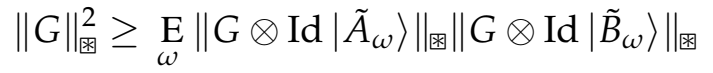

$$
\begin{aligned}
& \geq \underset{\omega}{\mathrm{E}} \underset{v \sim v^{\prime}}{\mathrm{E}} \sum_{b \leftrightarrow b^{\prime}}\left\langle\Gamma_{d d^{\prime}}\left|\overline{\tilde{A}_{\omega v}^{b}} \otimes \tilde{B}_{\omega v^{\prime}}^{b^{\prime}}\right| \Gamma_{d d^{\prime}}\right\rangle \\
& \geq \underset{\omega}{\mathrm{E}} \underset{v \sim v^{\prime}}{\mathrm{E}} \sum_{b \leftrightarrow b^{\prime}} \|\left|\Psi_{\omega v v}\right\rangle \|^{-2}\left\langle\Psi_{\omega v v^{\prime}}\right| \overline{U_{\omega v}^{+} \hat{A}_{\omega v}^{-1 / 2} \hat{A}_{\omega v}^{b} \hat{A}_{\omega v}^{-1 / 2} U_{\omega v}} \\
& \otimes U_{\omega v^{\prime}} \hat{A}_{\omega v^{\prime}}^{-1 / 2} \hat{A}_{\omega v^{\prime}}^{b^{\prime}} \hat{A}_{\omega v^{\prime}}^{-1 / 2} U_{\omega v^{\prime}}^{+}\left|\Psi_{\omega v v^{\prime}}\right\rangle-O\left(\eta^{1 / 12}\right) \\
& =\underset{\omega}{\mathrm{E}} \underset{v \sim v^{\prime}}{\mathrm{E}} \sum_{b \leftrightarrow b^{\prime}} \|\left|\Psi_{\omega v v}\right\rangle \|^{-2}\left\langle\hat{\Psi}\left|\overline{\hat{A}}_{\omega v}^{b} \otimes \hat{A}_{\omega v^{\prime}}^{b^{\prime}}\right| \hat{\Psi}\right\rangle-O\left(\eta^{1 / 12}\right) \text {, }
\end{aligned}
$$

where the second line uses the definition of $\tilde{A}_{\omega v}^{b}$ and (35) and the third is by definition of $\left|\Psi_{\omega v v^{\prime}}\right\rangle$. To conclude, note that applying Markov's inequality to (13) we get that a fraction at least $1-\eta^{1 / 3}$ of $v \sim v^{\prime}$ are such that

$$
\underset{\omega}{\mathrm{E}} \sum_{b \leftrightarrow b^{\prime}}\left\langle\hat{\Psi}\left|\overline{\hat{A}_{\omega v}^{b}} \otimes \hat{A}_{\omega v^{\prime}}^{b^{\prime}}\right| \hat{\Psi}\right\rangle \geq\left(1-\eta^{2 / 3}\right) \underset{\omega}{\mathrm{E}} \|\left|\Psi_{\omega v v}\right\rangle \|^{2},
$$


where here we crucially used the max on the right-hand side of (13) to allow ourselves use the same $v$ on the right-hand side as on the left-hand side. For any such $v \sim v^{\prime}$, a fraction $1-\eta^{1 / 3}$ of $\omega \in \Omega$ will be such that

$$
\sum_{b \leftrightarrow b^{\prime}}\left\langle\hat{\Psi}\left|\overline{\hat{A}_{\omega v}^{b}} \otimes \hat{A}_{\omega v^{\prime}}^{b^{\prime}}\right| \hat{\Psi}\right\rangle \geq\left(1-\eta^{1 / 3}\right) \|\left|\Psi_{\omega v v}\right\rangle \|^{2} .
$$

For these $v \sim v^{\prime}$ and $\omega$ the right-hand side of (36) is at least $1-\eta^{1 / 3}-O\left(\eta^{1 / 12}\right)$, and their total weight constitutes at least an $\left(1-2 \eta^{1 / 3}\right)$ fraction of the total.

\section{The correlated sampling lemma}

In this section we prove our quantum correlated sampling lemma.

Lemma 15. Let $d$ be an integer and $\delta>0$. There exists an integer $d^{\prime}$, and for every state $|\psi\rangle \in \mathbb{C}^{d} \otimes \mathbb{C}^{d}$ unitaries $V_{\psi}, W_{\psi}$ acting on $\mathbb{C}^{d d^{\prime}}$, such that the following holds for any two states $|\psi\rangle,|\varphi\rangle \in \mathbb{C}^{d} \otimes \mathbb{C}^{d}$ :

$$
\| \overline{V_{\psi}} \otimes W_{\varphi}\left|\Gamma_{d d^{\prime}}\right\rangle-|\psi\rangle\left|\Gamma_{d^{\prime}}\right\rangle\left\|=O\left(\max \left\{\delta^{1 / 12}, \||\psi\rangle-|\varphi\rangle \|^{1 / 6}\right\}\right)\right.
$$

where here $\left|\Gamma_{d}\right\rangle \propto \sum_{1 \leq i \leq d} i^{-1 / 2}|i\rangle|i\rangle$ is the (properly normalized) d-dimensional embezzlement state.

A variant of the lemma holding for the special case of $|\psi\rangle=|\varphi\rangle$ was shown in [vH03], where the "embezzlement state" $\left|\Gamma_{d}\right\rangle$ was first introduced. It is not hard to see however that the construction of the unitaries $V_{\psi}, W_{\varphi}$ given in that paper does not satisfy the conclusion of Lemma 15. For instance, if $|\psi\rangle=\sqrt{(1+\varepsilon) / 2}|00\rangle+\sqrt{(1-\varepsilon) / 2}|11\rangle$ and $|\varphi\rangle=\sqrt{(1-\varepsilon) / 2}|00\rangle+\sqrt{(1+\varepsilon) / 2}|11\rangle)$ then one can check that for any $\varepsilon>0$ the unitaries from [vH03] will be such that $\| \overline{V_{\psi}} \otimes W_{\varphi}\left|\Gamma_{2 d^{\prime}}\right\rangle-|\psi\rangle\left|\Gamma_{d^{\prime}}\right\rangle \| \geq 1 / 4$. This is due to the near-degeneracy in the spectrum of the reduced density matrices of $|\psi\rangle,|\varphi\rangle$; our proof of Lemma 15 shows that this is essentially the only obstacle that needs to be overcome in order to obtain a robust correlated sampling procedure.

Lemma 15 can be seen as a quantum analogue of Holenstein's correlated sampling lemma [Hol09], which played an important role in his proof of the classical parallel repetition theorem. There the players receive as inputs a description of a distribution $p, q$ respectively such that $\|p-q\|_{1}=\delta$. Their goal is to sample an element $u \sim p$ for the first player, $v \sim q$ for the second player, such that $u=v$ with probability $1-O(\delta)$. This task can be reproduced in our setting by giving the states $|\psi\rangle=\sum_{u} \sqrt{p(u)}|u\rangle|u\rangle$ to the first player and $|\phi\rangle=\sum_{v} \sqrt{q(v)}|v\rangle|v\rangle$ to the second. If the players run our procedure and then measure their joint state in the computational basis they will obtain samples with a distribution close to $p$ and $q$, and moreover these samples will be identical with high probability...though our proof would require them to use entanglement in order to do so!

We note that we have not tried to optimize the parameters appearing in the lemma. In particular, from our proof one can verify that taking $d^{\prime}=2^{O\left((d / \delta)^{2}\right)}$ in the lemma is sufficient, but this is probably far from optimal. Indeed, the method in [vH03] gives $d^{\prime}=d^{O(1 / \delta)}$; it may be possible to achieve such a polynomial dependence here as well.

Proof of Lemma 15 We define the unitaries $\overline{V_{\psi}}, W_{\varphi}$ implicitly through the following procedure, in which two players Alice, Bob receive classical descriptions of two bipartite states $|\psi\rangle,|\varphi\rangle$ respectively, each of local dimension $d$, as well as a precision parameter $\delta>0$. The unitaries $\overline{V_{\psi}}$ and $W_{\varphi}$ correspond to their respective local quantum operations as described in the procedure. The players' initial state consists of a classical description of the states $|\psi\rangle,|\varphi\rangle$ respectively (where each coefficient is specified with poly $\log \left(\delta, d^{-1}\right)$ 
bits of precision), a large supply of private qubits initialized in the $|0\rangle$ state, a large supply of shared EPR pairs that they will use as classical shared randomness, and an embezzlement state $\left|\Gamma_{d d^{\prime}}\right\rangle$ for some large enough $d^{\prime}$.

1. Let $d$ be the local dimension of $|\psi\rangle$ and $|\varphi\rangle, \delta$ the precision parameter given as part of the input, and $\eta>0$ a small parameter to be specified later.

2. Using shared randomness, the players jointly compute a sequence $\tau_{0}, \ldots, \tau_{K+1}$, where $K=\left\lceil\frac{\log (d / \delta)}{\log (1+\eta)}\right\rceil$, as follows. They set $\tau_{0}=1, \tau_{K+1}=0$, and for $k=1, \ldots, K$ they jointly sample $\tau_{k}$ uniformly at random in the interval $\left[(1+\eta)^{-k},(1+\eta)^{-k+1}\right)$.

3. Both players individually compute a classical description of the same (normalized) state

$$
\left|\xi_{0}\right\rangle \propto \sum_{k=0}^{K} \tau_{k}|k, k\rangle_{A B}\left|\Phi_{d}\right\rangle_{A B}
$$

where $\left|\Phi_{d}\right\rangle$ is the un-normalized maximally entangled state on $\mathbb{C}^{d} \otimes \mathbb{C}^{d}$. Let $N=\left\lceil\left(2 \delta d \sum_{k} \tau_{k}^{2}\right)^{-2}\right\rceil$. Alice and Bob jointly generate $N$ copies of $\left|\xi_{0}\right\rangle$, which they can achieve using the universal ambezzling procedure from [vH03] provided $d^{\prime}$ is large enough.

4. Alice (resp. Bob) computes the Schmidt decomposition $|\psi\rangle=\sum_{i} \lambda_{i}\left|u_{i}\right\rangle\left|u_{i}^{\prime}\right\rangle$ (resp. $|\varphi\rangle=\sum_{i} \mu_{i}\left|v_{i}\right\rangle\left|v_{i}^{\prime}\right\rangle$ ). She sets $S_{k}$ (resp. $T_{k}$ ) as the set of those indices $i$ such that $\lambda_{i} \in\left[\tau_{k+1}, \tau_{k}\right)\left(\right.$ resp. $\mu_{i} \in\left[\tau_{k+1}, \tau_{k}\right)$ ), $s_{k}=\left|S_{k}\right|$ (resp. $t_{k}=\left|T_{k}\right|$ ), and $P_{k}$ (resp. $Q_{k}$ ) the projector on the the span of the $\left|u_{i}\right\rangle$ for $i \in S_{k}$ (resp. $\left|v_{i}\right\rangle$ for $i \in T_{k}$ ).

5. Alice measures her share of the first copy of $\left|\xi_{0}\right\rangle$ using the two-outcome measurement $\left\{P_{A}, \operatorname{Id}-P_{A}\right\}$ where $P_{A}:=\sum_{k}|k\rangle\langle k| \otimes P_{k}$. Bob proceeds similarly with $P_{B}:=\sum_{k}|k\rangle\langle k| \otimes Q_{k}$. If either of them obtains the first outcome they proceed to the next step. Otherwise, they repeat this step with the next copy of $\left|\xi_{0}\right\rangle$ (assuming, but not knowing, that the other party also starts over). If all copies have been used they abort the protocol.

6. Alice (resp. Bob) controls on the second register of $\left|\xi_{0}\right\rangle$ to erase $|k\rangle$ in the first register. (This is possible since the $P_{k}$ (resp. $Q_{k}$ ) are orthogonal projections.) The players discard all qubits but the remaining register of $\left|\xi_{0}\right\rangle$.

Throughout the analysis we assume without loss of generality that $\delta \geq \||\psi\rangle-|\varphi\rangle \|^{2}$. We will show that with probability at least $1-O\left(\delta^{1 / 12}\right)$ the procedure described above results in a shared state between Alice and Bob that is within trace distance $O\left(\delta^{1 / 12}\right)$ of both $|\psi\rangle$ and $|\varphi\rangle$. Our first claim shows that, based on the $\tau_{k}$, the players can each compute a discretized version of their inputs that both have (a slightly re-scaled version of) the $\tau_{k}$ as Schmidt coefficients.

Claim 16. Define

$$
|\Psi\rangle:=C \sum_{k} \tau_{k} \sum_{i \in S_{k}}\left|u_{i}\right\rangle\left|u_{i}^{\prime}\right\rangle \quad \text { and } \quad|\Phi\rangle:=C^{\prime} \sum_{k} \tau_{k} \sum_{i \in T_{k}}\left|v_{i}\right\rangle\left|v_{i}^{\prime}\right\rangle,
$$

where the $\tau_{k}, S_{k}$ and $T_{k}$ are as defined in the protocol and $C, C^{\prime}$ are appropriate normalization constants. Then

$$
(1+\eta)^{-1} \leq C, C^{\prime} \leq 1
$$


and

$$
\max \left\{\||\psi\rangle-|\Psi\rangle\left\|^{2},\right\||\varphi\rangle-|\Phi\rangle \|^{2}\right\}=O(\eta)
$$

Proof. We have $C^{-2}=\sum_{k} \tau_{k}^{2} s_{k}$ which by definition of $S_{k}$ satisfies

$$
1=\sum_{i} \lambda_{i}^{2} \leq \sum_{k} \tau_{k}^{2} s_{k} \leq \sum_{i}(1+\eta)^{2} \lambda_{i}^{2} \leq(1+\eta)^{2} .
$$

A similar calculation holds for $C^{\prime}$, proving (37). Next we bound the first term in (38), the second being similar. Using the definition of $|\Psi\rangle$ and (37) we have

$$
\begin{aligned}
\||\psi\rangle-|\Psi\rangle \|^{2} & \leq \sum_{k} \sum_{i \in S_{k}}\left(\lambda_{i}-\tau_{k}\right)^{2}+O(\eta) \\
& \leq \sum_{k} \sum_{i \in S_{k}} \tau_{k}^{2}\left(1-\frac{1}{1+\eta}\right)^{2}+O(\eta) \\
& =O(\eta) .
\end{aligned}
$$

Our next claim shows that the subspaces $P_{k}, Q_{k}$ computed by the players are close, in the following sense.

Claim 17. The following holds with probability at least $1-O\left(\delta^{1 / 6} \eta^{-1 / 3}\right)$ over the choice of the $\tau_{k}$ :

$$
\sum_{k} \tau_{k}^{2} \operatorname{Tr}\left(P_{k} Q_{k}\right)=1-O\left(\delta^{1 / 6} \eta^{-1 / 3}\right)
$$

Proof. Using Claim 16 and $\||\psi\rangle-|\varphi\rangle \|^{2} \leq \delta$ we deduce that $|\langle\Phi \mid \Psi\rangle|^{2}=C C^{\prime} \sum_{k, k^{\prime}} \tau_{k} \tau_{k}^{\prime} \operatorname{Tr}\left(P_{k} Q_{k^{\prime}}\right)=$ $1-O(\eta)$. To prove the claim we bound the contribution of those terms for which $k \neq k^{\prime}$ :

$$
\begin{aligned}
\sum_{k \neq k^{\prime}} \tau_{k} \tau_{k^{\prime}} \operatorname{Tr}\left(P_{k} Q_{k^{\prime}}\right) & =\sum_{k \neq k^{\prime}} \tau_{k} \tau_{k^{\prime}} \sum_{i \in S_{k}} \sum_{j \in T_{k^{\prime}}}\left|\left\langle u_{i} \mid v_{j}\right\rangle\right|^{2} \\
& \leq(1+\eta)^{2}\left(\sum_{\substack{k \neq k^{\prime}, i \in S_{k}, j \in T_{k^{\prime}} \\
\left|\sqrt{\lambda_{i} / \mu_{j}}-\sqrt{\mu_{j} / \lambda_{i}}\right|^{2} \geq \xi}} \lambda_{i} \mu_{j}\left|\left\langle u_{i} \mid v_{j}\right\rangle\right|^{2}+\sum_{\substack{k \neq k^{\prime}, z, i \in S_{k}, j \in T_{k^{\prime}} \\
\left|\sqrt{\lambda_{i} / \mu_{j}}-\sqrt{\mu_{j} / \lambda_{i}}\right|^{2}<\xi}} \lambda_{i} \mu_{j}\left|\left\langle u_{i} \mid v_{j}\right\rangle\right|^{2}\right),
\end{aligned}
$$

where $\xi>0$ is a parameter to be fixed later. We bound each of the two terms inside the brackets in (40) separately. The first term is at most

$$
\begin{aligned}
\sum_{i, j} \lambda_{i} \mu_{j}\left|\left\langle u_{i} \mid v_{j}\right\rangle\right|^{2} & \leq \sum_{i, j} \frac{\left|\lambda_{i}-\mu_{j}\right|^{2}}{\xi}\left|\left\langle u_{i} \mid v_{j}\right\rangle\right|^{2} \\
& \leq \xi^{-1} \sum_{i, j}\left|\lambda_{i}\right|^{2} \geq \xi \lambda_{i} \mu_{j} \\
& \leq\left.\xi_{j}\right|^{2}\left|\left\langle u_{i} \mid v_{j}\right\rangle\right|^{2} \\
& \leq \delta \xi^{-1} .
\end{aligned}
$$


To bound the second term in (40), note first that provided $\xi$ is at most a small constant times $\eta$ necessarily $k^{\prime}=k+1$ or $k^{\prime}=k-1$; our choice of $\xi$ will satisfy this condition. Suppose $k^{\prime}=k-1$, the other case being similar. Fix $i, j$ such that $\left|\sqrt{\lambda_{i} / \mu_{j}}-\sqrt{\mu_{j} / \lambda_{i}}\right|^{2}<\xi$. This condition implies $\left|\lambda_{i}-\mu_{j}\right|^{2} \leq \xi \mu_{j} \lambda_{i} \leq$ $\xi(1+\eta)^{-3} \tau_{k}^{2}$. Since $\tau_{k}$ is chosen uniformly in an interval of length $\tau_{k} \eta(1+\eta)^{-1}$, the expected fraction of pairs $(i, j)$ such that such that $\left|\sqrt{\lambda_{i} / \mu_{j}}-\sqrt{\mu_{j} / \lambda_{i}}\right|^{2}<\xi$ and $\lambda_{i} \leq \tau_{k} \leq \mu_{j}$ is at most $O(\sqrt{\xi} / \eta)$. Hence, on expectation over the choice of the $\tau_{k}$ we have

$$
\sum_{\substack{k \neq k^{\prime}, i \in S_{k}, j \in T_{k^{\prime}} \\\left|\sqrt{\lambda_{i} / \mu_{j}}-\sqrt{\mu_{j} / \lambda_{i}}\right|^{2}<\xi}} \lambda_{i} \mu_{j}\left|\left\langle u_{i} \mid v_{j}\right\rangle\right|^{2} \leq O\left(\sqrt{\xi} \eta^{-1}\right) \sum_{i, j} \lambda_{i} \mu_{j}\left|\left\langle u_{i} \mid v_{j}\right\rangle\right|^{2}=O\left(\sqrt{\xi} \eta^{-1}\right) .
$$

Choosing $\xi=(\delta \eta)^{2 / 3}$, we obtain that (39) holds, on expectation over the choice of the $\tau_{k}$, with a right-hand side of $1-O\left(\delta^{1 / 3} \eta^{-2 / 3}\right)$. (The condition that $\xi \ll \eta$ is equivalent to $\delta \ll \eta^{1 / 3}$, which we may assume holds without loss of generality, as otherwise the bound in the claim is trivial.) The left-hand side is at most 1 , and applying Markov's inequality proves the claim.

Our last claim analyzes the outcome of the sampling procedure, proving the lemma.

Claim 18. Let $|\psi\rangle,|\varphi\rangle$ be such that $\||\psi\rangle-|\varphi\rangle \|^{2} \leq \delta$, and set $\eta=\delta^{1 / 4}$. With probability at least $1-O\left(\delta^{1 / 12}\right)$, the sampling procedure described above terminates with Alice and Bob in a shared state $|\xi\rangle$ such that $\||\xi\rangle-|\psi\rangle \|^{2}=O\left(\delta^{1 / 12}\right)$.

Proof. Suppose first that (39) holds and that Alice and Bob both proceed to the third step synchronously. In that case, at the end of the procedure their joint state is

$$
|\xi\rangle:=C^{\prime \prime} \sum_{k} \tau_{k} \sum_{i \in S_{k}, j \in T_{k}}\left\langle u_{i} \mid v_{j}\right\rangle\left|u_{i}\right\rangle\left|v_{j}\right\rangle,
$$

where the normalization constant $C^{\prime \prime}$ satisfies

$$
\left(C^{\prime \prime}\right)^{-2}=\sum_{k} \tau_{k}^{2} \sum_{i \in S_{k}, j \in T_{k}}\left|\left\langle u_{i} \mid v_{j}\right\rangle\right|^{2}=\sum_{k} \tau_{k}^{2} \operatorname{Tr}\left(P_{k} Q_{k}\right)=1-O\left(\eta+\delta^{1 / 3} \eta^{-2 / 3}\right)
$$

by Claim 17 We can thus evaluate the overlap of $|\xi\rangle$ with $|\Phi\rangle$ as

$$
\begin{aligned}
\langle\xi \mid \Phi\rangle & \geq \sum_{k} \tau_{k}^{2} \sum_{i \in S_{k}, j \in T_{k}}\left|\left\langle u_{i} \mid v_{j}\right\rangle\right|^{2}-O\left(\delta^{1 / 3} \eta^{-2 / 3}\right) \\
& =1-O\left(\delta^{1 / 6} \eta^{-1 / 3}\right),
\end{aligned}
$$

where for the first equality we used orthogonality of the $\left|u_{i}\right\rangle$, and the last again follows from Claim 17 .

Next we compute the probability that in the second step Alice and Bob both obtain the first outcome of their respective POVM in the same iteration. The probability that Alice alone obtains a successful outcome is $\sum_{k} \tau_{k}^{2} s_{k} /\left(d \sum_{k} \tau_{k}^{2}\right)=(1+\Theta(\eta))\left(d \sum_{k} \tau_{k}^{2}\right)^{-1}$ by (37). The same holds for Bob. With probability at least $1-\delta^{2}$, both of them obtain a successful outcome before the number $N$ of copies of $\left|\xi_{0}\right\rangle$ runs out. Moreover, the probability that they simultaneously obtain the first outcome is

$$
\left(d \sum_{k} \tau_{k}^{2}\right)^{-1} \sum_{k} \tau_{k}^{2} \operatorname{Tr}\left(P_{k} Q_{k}\right) \geq\left(1-O\left(\delta^{1 / 6} \eta^{-1 / 3}\right)\right)\left(d \sum_{k} \tau_{k}^{2}\right)^{-1}
$$

by Claim 17 Hence the probability that they simultaneously proceed to the third step of the protocol is at least $1-O\left(\delta^{1 / 6} \eta^{-1 / 3}\right)$. Choosing $\eta=\delta^{1 / 4}$ proves the lemma. 


\section{A Auxiliary claims}

Claim 19. Let $X \in \mathcal{L}\left(\mathbb{C}^{d}\right), Y \in \mathcal{L}\left(\mathbb{C}^{d^{\prime}}\right)$ be two operators and $|\Psi\rangle \in \mathbb{C}^{d} \otimes \mathbb{C}^{d^{\prime}}$ a bipartite state with Schmidt decomposition $|\Psi\rangle=\sum_{i} \lambda_{i}\left|u_{i}\right\rangle\left|v_{i}\right\rangle$, where the $\lambda_{i}$ are non-negative reals. Then

$$
\langle\Psi|X \otimes Y| \Psi\rangle=\operatorname{Tr}\left(X K Y^{T} K^{\dagger}\right),
$$

where $K=\sum_{i} \lambda_{i}\left|u_{i}\right\rangle\left\langle v_{i}\right|$ and the transpose is taken in the bases specified by the $\left|u_{i}\right\rangle$ and $\left|v_{j}\right\rangle$. In particular, if $\left|u_{i}\right\rangle=\left|v_{i}\right\rangle$ for every $i, K$ is positive semidefinite and (41) evaluates to $\operatorname{Tr}\left(X K Y^{T} K\right)$.

Proof. The proof follows by direct calculation, expanding the left-hand side of (41) using the Schmidt decomposition of $|\Psi\rangle$ and the right-hand side using the definition of $K$.

Claim 20. For any $d$ and operators $A_{i} \in \mathcal{L}\left(\mathbb{C}^{d}\right), B_{i} \in \mathcal{L}\left(\mathbb{C}^{d^{\prime}}\right)$,

$$
\left\|\sum_{i} \overline{A_{i}} \otimes B_{i}\right\|_{\infty}^{2} \leq\left\|\sum_{i} \overline{A_{i}} \otimes A_{i}\right\|_{\infty}\left\|\sum_{i} \overline{B_{i}} \otimes B_{i}\right\|_{\infty}
$$

Proof. Let $|\Psi\rangle \in \mathbb{C}^{d} \otimes \mathbb{C}^{d}$ be a unit vector with Schmidt decomposition $|\Psi\rangle=\sum_{i} \lambda_{i}\left|u_{i}\right\rangle\left|v_{i}\right\rangle$ and $K=$ $\sum_{i} \lambda_{i}\left|u_{i}\right\rangle\left\langle v_{i}\right|$. Using cyclicity of the trace,

$$
\operatorname{Tr}\left(A_{i} K B_{i}^{\dagger} K^{\dagger}\right)=\operatorname{Tr}\left(\left(\left(K^{\dagger} K\right)^{-1 / 4} K^{\dagger} A_{i}\left(K K^{\dagger}\right)^{1 / 4}\right)\left(\left(K K^{\dagger}\right)^{-1 / 4} K B_{i}^{\dagger}\left(K^{\dagger} K\right)^{1 / 4}\right)\right),
$$

and the Cauchy-Schwarz inequality for the trace inner-product gives

$$
\left|\operatorname{Tr}\left(A_{i} K B_{i}^{\dagger} K^{\dagger}\right)\right|^{2} \leq \operatorname{Tr}\left(A_{i} \sqrt{K K^{\dagger}} A_{i}^{\dagger} \sqrt{K K^{\dagger}}\right) \operatorname{Tr}\left(B_{i}^{\dagger} \sqrt{K^{\dagger} K} B_{i} \sqrt{K^{\dagger} K}\right) .
$$

Using Claim 19 we may then write

$$
\begin{aligned}
& \left|\left\langle\Psi\left|\left(\sum_{i} \overline{A_{i}} \otimes B_{i}\right)\right| \Psi\right\rangle\right|=\left|\sum_{i} \operatorname{Tr}\left(A_{i} K B_{i}^{\dagger} K^{\dagger}\right)\right| \\
& \leq \sum_{i} \sqrt{\operatorname{Tr}\left(A_{i} \sqrt{K K^{\dagger}} A_{i}^{\dagger} \sqrt{K K^{\dagger}}\right)} \sqrt{\operatorname{Tr}\left(B_{i}^{\dagger} \sqrt{K^{\dagger} K} B_{i} \sqrt{K^{\dagger} K}\right)} \\
& \leq\left(\sum_{i} \operatorname{Tr}\left(A_{i} \sqrt{K K^{\dagger}} A_{i}^{\dagger} \sqrt{K K^{\dagger}}\right)\right)^{1 / 2}\left(\sum_{i} \operatorname{Tr}\left(B_{i}^{\dagger} \sqrt{K^{\dagger} K} B_{i} \sqrt{K^{\dagger} K}\right)\right)^{1 / 2} \\
& =\left|\left\langle\Psi_{L}\left|\left(\sum_{i} \overline{A_{i}} \otimes A_{i}\right)\right| \Psi_{L}\right\rangle\right|^{1 / 2}\left|\left\langle\Psi_{R}\left|\left(\sum_{i} \overline{B_{i}} \otimes B_{i}\right)\right| \Psi_{R}\right\rangle\right|^{1 / 2},
\end{aligned}
$$

where for the first inequality we used (42), the second follows from the Cauchy-Schwarz inequality, and for the last we introduced $\left|\Psi_{L}\right\rangle=\sum_{i} \lambda_{i}\left|u_{i}\right\rangle\left|u_{i}\right\rangle,\left|\Psi_{R}\right\rangle=\sum_{i} \lambda_{i}\left|v_{i}\right\rangle\left|v_{i}\right\rangle$, and we used Claim 19 to re-write the expressions. Since (43) holds for any $|\Psi\rangle$, the claim is proved.

We next prove Claim 3, introduced in Section 2.2.

Claim 3 There exists a polynomial-time computable transformation mapping any two-player one-round game $G$ to a projection game $G^{\prime}$ such that the following hold:

$$
1-2\left(1-\operatorname{VAL}\left(G^{\prime}\right)\right) \leq \operatorname{VAL}(G) \leq \operatorname{VAL}\left(G^{\prime}\right)
$$


In particular, $\operatorname{VAL}\left(G^{\prime}\right)=1$ if and only if $\operatorname{VAL}(G)=1$, and $1-\operatorname{VAL}\left(G^{\prime}\right)=\Theta(1-\operatorname{VAL}(G))$. Moreover, for the entangled value we have the weaker bound

$$
\operatorname{VAL}^{*}\left(G^{\prime}\right) \leq \sqrt{\frac{1+\operatorname{VAL}^{*}(G)}{2}}
$$

which implies $1-\mathrm{VAL}^{*}\left(G^{\prime}\right)=\Omega\left(1-\mathrm{VAL}^{*}(G)\right)$.

Proof. Let $G$ be a game with (without loss of generality disjoint) question sets $\mathcal{U}, \mathcal{V}$, answer sets $\mathcal{A}, \mathcal{B}$, distribution on questions $\mu$ and acceptance predicate $V$. Let $G^{\prime}$ be the projection game corresponding to the following scenario. The referee selects a pair of questions $(u, v)$ at random from $\mu$, which it sends to the second player, and then sends either $u$ or $v$ to the first player, each with probability $1 / 2$. Formally, $G^{\prime}$ is defined by question sets $\mathcal{U}^{\prime}=\mathcal{U} \cup \mathcal{V}, \mathcal{V}^{\prime}=\mathcal{U} \times \mathcal{V}$, answer sets $\mathcal{A}^{\prime}=\mathcal{A} \cup \mathcal{B}, \mathcal{B}^{\prime}=\mathcal{A} \times \mathcal{B}$, and a distribution $\mu^{\prime}$ given by $\mu^{\prime}(u,(u, v))=\mu^{\prime}(u, v) / 2, \mu^{\prime}(v,(u, v))=\mu^{\prime}(u, v) / 2$, and 0 otherwise. For any $(u, v)$ and $(a, b)$ let $\pi_{u,(u, v)}$ be such that $\pi_{u,(u, v)}(a, b)=a$ and $\pi_{v,(u, v)}(a, b)=b$ if $V(a, b, u, v)=1$, and there is no valid answer for the first player if the second player's answers are such that $V(a, b, u, v)=0$.

Then clearly $G^{\prime}$ is a projection game. Let $|f\rangle,|g\rangle$ be classical strategies for the players such that $\operatorname{vaL}(G,|f\rangle,|g\rangle)=\operatorname{vaL}(G)$. Consider the strategy $\left(\left|f^{\prime}\right\rangle,\left|g^{\prime}\right\rangle\right)$ for $G^{\prime}$ in which $\left|f^{\prime}\right\rangle$ answers as $|f\rangle$ to questions $u \in \mathcal{U}$ and as $|g\rangle$ to questions $v \in \mathcal{V}$, and $\left|g^{\prime}\right\rangle$ answers as $(|f\rangle,|g\rangle)$. Then whenever the strategy $(|f\rangle,|g\rangle)$ provides answers to a pair of questions $(u, v)$ that satisfy the predicate $V$ the strategy $(|f\rangle,|g\rangle)$ gives answers to both $(u,(u, v))$ and $(v,(u, v))$ that are accepted in $G^{\prime}$, hence

$$
\operatorname{VAL}\left(G^{\prime}\right) \geq \operatorname{VAL}\left(G^{\prime},\left|f^{\prime}\right\rangle,\left|g^{\prime}\right\rangle\right) \geq \operatorname{VAL}(G,|f\rangle,|g\rangle)=\operatorname{VAL}(G) \text {. }
$$

Conversely, let $\left(\left|f^{\prime}\right\rangle,\left|g^{\prime}\right\rangle\right)$ be a strategy for $G^{\prime}$ such that $\operatorname{vAL}\left(G^{\prime}\right)=\operatorname{VAL}\left(G^{\prime},\left|f^{\prime}\right\rangle,\left|g^{\prime}\right\rangle\right)$. Decompose $\left|f^{\prime}\right\rangle$ into a pair of strategies $|f\rangle,|g\rangle$ in $G$, depending on whether the question is $u \in \mathcal{U}$ or $v \in \mathcal{V}$. The pair $(|f\rangle,|g\rangle)$ will give a rejected answer to a pair of questions $(u, v)$ only if $\left(\left|f^{\prime}\right\rangle,\left|g^{\prime}\right\rangle\right)$ gave a rejected answer to at least one of the questions $(u,(u, v))$ and $(v,(u, v))$ in $G^{\prime}$. In the worst case the $\left(1-\operatorname{vaL}\left(G^{\prime},|f\rangle,|g\rangle\right)\right)$ probability that $\left(\left|f^{\prime}\right\rangle,\left|g^{\prime}\right\rangle\right)$ provides rejected answers in $G^{\prime}$ is, say, fully concentrated on questions of the form $(u,(u, v))$. Hence

$$
\operatorname{VAL}(G) \geq \operatorname{VAL}(G,|f\rangle,|g\rangle) \geq 1-2\left(1-\operatorname{vaL}\left(G^{\prime},\left|f^{\prime}\right\rangle,\left|g^{\prime}\right\rangle\right)\right)=1-2\left(1-\operatorname{vaL}\left(G^{\prime}\right)\right) .
$$

Finally, let $\left(\left|A^{\prime}\right\rangle,\left|B^{\prime}\right\rangle\right)$ be a pair of quantum strategies such that $\operatorname{VAL}^{*}\left(G^{\prime}\right)=\operatorname{VAL}^{*}\left(G^{\prime},\left|A^{\prime}\right\rangle,\left|B^{\prime}\right\rangle\right)$. To $|A\rangle$ are unambiguously associated measurement operators $\left\{A_{u}^{a}\right\}_{a}$ for every $u \in \mathcal{U}$, and $\left\{A_{v}^{b}\right\}_{b}$ for $v \in \mathcal{V}$. Hence

$$
\begin{aligned}
\operatorname{VAL}^{*}\left(G^{\prime}\right) & =\left\|\underset{u \sim v}{\mathrm{E}} \frac{1}{2} \sum_{(a, b): V(a, b, u, v)=1} \overline{A_{u}^{a}} \otimes B_{u, v}^{a, b}+\overline{A_{v}^{b}} \otimes B_{u, v}^{a, b}\right\|_{\infty} \\
& \leq\left\|{\underset{u \sim v}{\mathrm{E}} \frac{1}{4}}_{(a, b): V(a, b, u, v)=1} \overline{\left(A_{u}^{a}+A_{v}^{b}\right)} \otimes\left(A_{u}^{a}+A_{v}^{b}\right)\right\|_{\infty}^{1 / 2}\left\|_{u \sim v} \sum_{(a, b): V(a, b, u, v)=1} \overline{B_{u, v}^{a, b}} \otimes B_{u, v}^{a, b}\right\|_{\infty}^{1 / 2} \\
& \left.\leq\left(\frac{1}{2}+\frac{1}{2} \|{\underset{u \sim v}{\mathrm{E}}}_{(a, b): V(a, b, u, v)=1} \overline{A_{u}^{a}} \otimes A_{v}^{b}\right) \|_{\infty}\right)^{1 / 2},
\end{aligned}
$$

where the last inequality uses the triangle inequality for the operator norm and the fact that $\left\|\sum_{i} X_{i} \otimes Y_{i}\right\|_{\infty}=$ $\left\|\sum_{i} Y_{i} \otimes X_{i}\right\|_{\infty}$ for any $X_{i}, Y_{i}$. Hence the pair of strategies $\left(\left|A_{\mid \mathcal{U}}\right\rangle,\left|A_{\mid \mathcal{V}}\right\rangle\right)$ for $G$ achieves a value at least

$$
\operatorname{VAL}^{*}(G) \geq \operatorname{VAL}^{*}\left(G,\left|A_{\mid \mathcal{U}}\right\rangle,\left|A_{\mid \mathcal{V}}\right\rangle\right) \geq 2 \operatorname{VAL}^{*}\left(G^{\prime}\right)^{2}-1
$$

as claimed. 


\section{References}

[AGR81] A. Aspect, P. Grangier, and G. Roger. Experimental tests of realistic local theories via Bell's theorem. Phys. Rev. Lett., 47(7):460-463, 1981.

[AHW00] G. G. Amosov, A. S. Holevo, and R. F. Werner. On some additivity problems in quantum information theory. Technical report, arXiv:math-ph/0003002, 2000.

[Ara02] P. K. Aravind. The magic squares and Bell's theorem. Technical report, arXiv:quant$\mathrm{ph} / 0206070,2002$.

[BBLV12] J. Briët, H. Buhrman, T. Lee, and T. Vidick. Multipartite entanglement in XOR games. Quantum Information and Computation, 2012.

[Bel64] J. S. Bell. On the Einstein-Podolsky-Rosen paradox. Physics, 1:195-200, 1964.

[BK02] H. Barnum and E. Knill. Reversing quantum dynamics with near-optimal quantum and classical fidelity. J. Math. Physics, 43(5):2097-2106, 2002.

[BRR ${ }^{+}$09] B. Barak, A. Rao, R. Raz, R. Rosen, and R. Shaltiel. Strong parallel repetition theorem for free projection games. In Approximation, Randomization, and Combinatorial Optimization. Algorithms and Techniques, volume 5687, pages 352-365. Springer Berlin Heidelberg, 2009.

[CHSH69] J. F. Clauser, M. A. Horne, A. Shimony, and R. A. Holt. Proposed experiment to test local hidden-variable theories. Phys. Rev. Lett., 23:880-884, 1969.

[CJPP11] T. Cooney, M. Junge, C. Palazuelos, and D. Pérez-García. Rank-one quantum games. Technical report, arXiv:1112.3563, 2011.

[CSUU08] R. Cleve, W. Slofstra, F. Unger, and S. Upadhyay. Perfect parallel repetition theorem for quantum XOR proof systems. Comput. Complexity, 17(2):282-299, 2008.

[Din07] I. Dinur. The pcp theorem by gap amplification. J. ACM, 54(3), June 2007.

[DS13] I. Dinur and D. Steurer. Analytical approach to parallel repetition. Technical report, arXiv:1305.1979, 2013.

[EPR35] A. Einstein, B. Podolsky, and N. Rosen. Can quantum-mechanical description of physical reality be considered complete? Physical Review, 47:777-780, 1935.

[Fei91] U. Feige. On the success probability of two provers in one-round proof systems. In Proc. 6th IEEE Structure in Complexity Theory, pages 116-123. 1991.

[FK00] U. Feige and J. Kilian. Two-Prover Protocols—Low Error at Affordable Rates. SIAM J. Comput., 30(1):324, 2000.

[FL92] U. Feige and L. Lovász. Two-prover one-round proof systems: Their power and their problems. In Proceedings of the 24th Annual ACM Symposium on Theory of Computing (STOC), pages 733-744. 1992.

[FRS88] L. Fortnow, J. Rompel, and M. Sipser. On the power of multi-prover interactive protocols. In Theoretical Computer Science, pages 156-161. 1988. 
[FvdG99] C. A. Fuchs and J. van de Graaf. Cryptographic distinguishability measures for quantummechanical states. IEEE Transactions on Information Theory, 45(4):1216-1227, 1999.

[Has09] M. B. Hastings. Superadditivity of communication capacity using entangled inputs. Nature Physics, 5(4):255-257, 2009.

[HJS ${ }^{+}$96] P. Hausladen, R. Jozsa, B. Schumacher, M. Westmoreland, and W. K. Wootters. Classical information capacity of a quantum channel. Phys. Rev. A, 54:1869, 1996.

[Hol09] T. Holenstein. Parallel repetition: Simplification and the no-signaling case. Theory of Computing, 5(1):141-172, 2009.

[HR09] E. Hänggi and R. Renner. Device-independent quantum key distribution with commuting measurements. Technical report, arXiv:1009.1833, 2009.

[HW94] P. Hausladen and W. K. Wootters. A 'pretty good' measurement for distinguishing quantum states. J. Modern Optics, 41(12):2385-2390, 1994.

[HW08] P. Hayden and A. Winter. Counterexamples to the maximal p-norm multiplicativity conjecture for all $p>$ 1. Comm. Math. Phys., 284(1):263-280, 2008.

[IV12] T. Ito and T. Vidick. A multi-prover interactive proof for NEXP sound against entangled provers. Proc. 53rd FOCS, pages 243-252, 2012.

$\left[\mathrm{JPPG}^{+} 10\right]$ M. Junge, C. Palazuelos, D. Pérez-García, I. Villanueva, and M. M. Wolf. Operator space theory: A natural framework for Bell inequalities. Physical Review Letters, 104:170405, 2010.

[Kit86] F. Kittaneh. Inequalities for the schatten p-norm. iv. Comm. Math. Phys., 106(4):581-585, 1986.

[KR10] J. Kempe and O. Regev. No strong parallel repetition with entangled and non-signaling provers. In Proc. 25th IEEE Conf. on Computational Complexity (CCC'10), pages 7-15. IEEE Computer Society, Washington, DC, USA, 2010.

[KV11] J. Kempe and T. Vidick. Parallel repetition of entangled games. In Proc. 43rd STOC, pages 353-362. 2011.

[MPA11] L. Masanes, S. Pironio, and A. Acín. Secure device-independent quantum key distribution with causally independent measurement devices. Nature Communications, 2(238):7, 2011.

[Rao08] A. Rao. Parallel repetition in projection games and a concentration bound. In Proc. 40th STOC, pages 1-10. ACM, 2008.

[Raz98] R. Raz. A parallel repetition theorem. SIAM J. Comput., 27:763-803, 1998.

[Raz08] R. Raz. A Counterexample to Strong Parallel Repetition. In 49th Annual IEEE Symposium on Foundations of Computer Science, pages 369-373. 2008.

[RR12] R. Raz and R. Rosen. A strong parallel repetition theorem for projection games on expanders. In Proc. 27th IEEE Conf. on Computational Complexity (CCC'12), pages 247-257. 2012. 
[TFKW13] M. Tomamichel, S. Fehr, J. Kaniewski, and S. Wehner. A monogamy-of-entanglement game with applications to device-independent quantum cryptography. New Journal of Physics, 15(10):103002, 2013.

[Ver94] O. Verbitsky. Towards the parallel repetition conjecture. Proceedings of IEEE 9th Annual Conference on Structure in Complexity Theory, pages 304-307, 1994.

[vH03] W. van Dam and P. Hayden. Universal entanglement transformations without communication. Phys. Rev. A, 67:060302(R), 2003.

[Vid13] T. Vidick. Three-player entangled XOR games are NP-hard to approximate. In Proc. 54th FOCS. 2013. 